\title{
NSU
}

Florida

NOVA SOUTHEASTERN

UNIVERSITY

Nova Southeastern University

NSUWorks

$2-2011$

\section{Finite Basis Problem for 2-Testable Monoids}

Edmond W. H. Lee

Simon Fraser University, edmond.lee@nova.edu

Follow this and additional works at: https://nsuworks.nova.edu/math_facarticles

Part of the Mathematics Commons

\section{NSUWorks Citation}

Lee, Edmond W. H., "Finite Basis Problem for 2-Testable Monoids" (2011). Mathematics Faculty Articles. 2. https://nsuworks.nova.edu/math_facarticles/2

This Article is brought to you for free and open access by the Department of Mathematics at NSUWorks. It has been accepted for inclusion in Mathematics Faculty Articles by an authorized administrator of NSUWorks. For more information, please contact nsuworks@nova.edu. 


\title{
Finite basis problem for 2-testable monoids
}

\author{
Edmond W. H. Lee ${ }^{1 *}$ \\ 1 Department of Mathematics, Simon Fraser University, British Columbia, Canada
}

Received 31 May 2010; accepted 26 October 2010

\begin{abstract}
A monoid $S^{1}$ obtained by adjoining a unit element to a 2-testable semigroup $S$ is said to be 2-testable. It is shown that a 2-testable monoid $S^{1}$ is either inherently non-finitely based or hereditarily finitely based, depending on whether or not the variety generated by the semigroup $S$ contains the Brandt semigroup of order five. Consequently, it is decidable in quadratic time if a finite 2-testable monoid is finitely based.

MSC: $\quad 20 \mathrm{M} 07$

Keywords: Semigroups $\cdot$ Monoids $\cdot$ Varieties $\cdot$ Finitely based $\cdot$ Hereditarily finitely based

(C) Versita Sp. z o.o.
\end{abstract}

\section{Introduction}

In the present article, all varieties are varieties of semigroups, that is, classes of semigroups that are closed under the formation of homomorphic images, subsemigroups, and arbitrary direct products. A variety is finitely based if its identities are finitely axiomatizable. A semigroup is finitely based if it generates a finitely based variety. The finite basis problem asks when a given finite semigroup is finitely based. This problem has been intensely investigated as early as the 1960s and is still open. Refer to the surveys of Shevrin and Volkov [20] and Volkov [27] for more information on semigroup varieties and the finite basis problem.

It is well known that the idempotent-generated 0-simple semigroup

$$
A_{2}=\left\langle a, b \mid a^{2}=a b a=a, b^{2}=0, b a b=b\right\rangle
$$

of order five plays several important roles in the theory of semigroup varieties. For instance, Trahtman [25] showed that the variety $A_{2}$ generated by the semigroup $A_{2}$ coincides with the class of 2-testable semigroups, that is, semigroups that

*E-mail: ewl@sfu.ca 
satisfy any identity formed by a pair of words that begin with the same letter, end with the same letter, and share the same set of factors of length two. The variety $A_{2}$ also coincides with the variety generated by all aperiodic 0 -simple semigroups [5] and is essential in the recent discovery and description of a new infinite series of limit varieties [16].

The semigroup $A_{2}$ is also an important example that is related to semigroups with very extreme and contrasting equational properties. By the early 1980s, Trahtman $[22,24]$ had proven that the semigroup $A_{2}$ is finitely based by the identities

$$
x^{3} \approx x^{2}, \quad x y x y x \approx x y x, \quad x y x z x \approx x z x y x
$$

Recently, the semigroup $A_{2}$ was shown to satisfy the stronger property of being hereditarily finitely based [9], that is, every semigroup in the variety $A_{2}$ is finitely based. On the other hand, the semigroup $A_{2}$ can be used to construct non-finitely based semigroups. Volkov [26] demonstrated that the direct product of the semigroup $A_{2}$ with any finite group is non-finitely based. Trahtman [23] proved that the monoid $A_{2}^{1}$ obtained from $A_{2}$ by adjoining a unit element is non-finitely based, and Sapir [19] even proved that $A_{2}^{1}$ is inherently non-finitely based in the sense that any locally finite variety containing it is non-finitely based.

Since the variety $A_{2}$ coincides with the class of 2-testable semigroups [25], it is reasonable to refer to a monoid $S^{1}$ as a 2-testable monoid whenever $S$ is a semigroup from $A_{2}$. Motivated by the contrasting equational properties of the semigroups $A_{2}$ and $A_{2}^{1}$, the present article is an in-depth investigation of the finite basis problem for 2-testable monoids. The non-finitely based monoid $A_{2}^{1}$ is vacuously 2-testable. It is routine to verify that the Brandt semigroup

$$
B_{2}=\left\langle c, d \mid c^{2}=d^{2}=0, c d c=c, d c d=d\right\rangle
$$

of order five satisfies the identities (1) and so belongs to the variety $A_{2}$. Therefore the monoid $B_{2}^{1}$ is also 2 -testable; this monoid is not only non-finitely based [18] but is also inherently non-finitely based [19].

\section{Remark 1.1.}

Up to isomorphism and anti-isomorphism, $A_{2}^{1}$ and $B_{2}^{1}$ are the only monoids that are minimal with respect to being nonfinitely based [14]. Consequently, the semigroups $A_{2}$ and $B_{2}$ are the smallest possible examples of a finitely based semigroup $S$ for which the monoid $S^{1}$ is non-finitely based.

For any semigroup or monoid $S$, let $\operatorname{VAR}\{S\}$ denote the semigroup variety generated by $S$. It is easy to show that if $S$ and $T$ are semigroups such that $S \in \operatorname{VaR}\{T\}$, then $S^{1} \in \operatorname{VAR}\left\{T^{1}\right\}$; see also Almeida [1, Lemma 7.1.1]. Since the monoid $B_{2}^{1}$ is inherently non-finitely based [19], a 2-testable monoid $S^{1}$ is also inherently non-finitely based whenever $B_{2} \in \operatorname{VAR}\{S\}$. It is thus natural to examine the finite basis problem for 2-testable monoids $S^{1}$ for which $B_{2} \notin V_{A R}\{S\}$. The main goal of the present article is to show that such monoids must be hereditarily finitely based, thereby establishing a dichotomy for 2-testable monoids with respect to the finite basis property.

\section{Theorem 1.2.}

Let $S$ be any semigroup in the variety $\mathrm{A}_{2}$.

(i) If $B_{2} \in \operatorname{VAR}\{S\}$, then the monoid $S^{1}$ is inherently non-finitely based.

(ii) If $B_{2} \notin \operatorname{VAR}\{S\}$, then the monoid $S^{1}$ is hereditarily finitely based.

Consequently, any 2-testable monoid is either inherently non-finitely based or hereditarily finitely based.

\section{Remark 1.3.}

(i) For any semigroup $S$ in the variety $A_{2}$, the semigroup $B_{2}$ belongs to the variety $V_{A R}\{S\}$ if and only if $S$ does not satisfy the identity $x y^{2} x \approx x y x$ [6]. Therefore, in the presence of Theorem 1.2, checking the finite basis property of a finite 2-testable monoid $S^{1}$ is a problem of complexity $O\left(|S|^{2}\right)$.

(ii) Varieties generated by 2-testable monoids will be identified in a sequel article [13].

There are six sections in the present article. Notation and background material are given in Section 2. Main arguments of the proof of Theorem 1.2(ii) are given in Section 3, while the finer details are deferred to Sections 4-6. 


\section{Preliminaries}

Most of the notation and background material of this article are given in this section. Refer to the monograph of Burris and Sankappanavar [2] for more information on universal algebra.

\subsection{Letters and words}

Let $\mathcal{X}$ be a fixed countably infinite alphabet throughout. Denote by $\mathcal{X}^{+}$and $\mathcal{X}^{*}$ the free semigroup and the free monoid over $\mathcal{X}$ respectively. Elements of $\mathcal{X}$ and $\mathcal{X}^{*}$ are referred to as letters and words respectively.

Let $x$ be any letter and $\boldsymbol{w}$ be any word. Then

- the content of $\mathbf{w}$, denoted by con (w), is the set of letters occurring in $\mathbf{w}$;

- the head of $\mathbf{w}$, denoted by $\mathrm{h}(\mathbf{w})$, is the first letter occurring in $\mathbf{w}$;

- the tail of $\mathbf{w}$, denoted by $\mathrm{t}(\mathbf{w})$, is the last letter occurring in $\mathbf{w}$;

- the initial part of $\mathbf{w}$, denoted by ini $(\mathbf{w})$, is the word obtained from $\mathbf{w}$ by retaining the first occurrence of each letter;

- the final part of $\mathbf{w}$, denoted by fin $(\mathbf{w})$, is the word obtained from $\mathbf{w}$ by retaining the last occurrence of each letter;

- $x$ is simple in $\mathbf{w}$ if $x$ occurs exactly once in $\mathbf{w}$;

- $\mathbf{w}$ is simple if any letter occurs at most once in $\mathbf{w}$;

- $\mathbf{w}$ is quadratic if any letter occurs at most twice in $\mathbf{w}$.

Note that by definition, the empty word is simple and any simple word is quadratic.

Let $\mathbf{w}$ be any quadratic word. If $\mathbf{w}=\mathbf{a} x \mathbf{b} x \mathbf{c}$ for some $x \in \mathcal{X}$ and $\mathbf{a}, \mathbf{b}, \mathbf{c} \in \mathcal{X}^{*}$ with $x \notin$ con (abc), then the distance between the two occurrences of $x$ in $\mathbf{w}$ is the length of $\mathbf{b}$. If $x_{1}, \ldots, x_{r}$ are all the non-simple letters of $\mathbf{w}$, then the separation degree of $\mathbf{w}$ is the sum $d_{1}+\cdots+d_{r}$, where $d_{i}$ is the distance between the two occurrences of $x_{i}$ in $\mathbf{w}$.

\subsection{Identities and varieties}

An identity is written as $\mathbf{u} \approx \mathbf{v}$ where $\mathbf{u}, \mathbf{v} \in \mathcal{X}^{+}$. A semigroup $S$ satisfies an identity $\mathbf{u} \approx \mathbf{v}$ if for any substitution $\varphi$ from $\mathcal{X}$ into $S$, the elements $\mathrm{u} \varphi$ and $\mathrm{v} \varphi$ of $S$ coincide. A variety $\mathrm{V}$ satisfies an identity $\mathbf{u} \approx \mathrm{v}$ if every semigroup in $\mathrm{V}$ satisfies $\mathbf{u} \approx \mathbf{v}$; this is indicated by $\mathbf{V} \vDash \mathbf{u} \approx \mathbf{v}$.

Let $\Sigma$ be any set of identities. An identity $\mathbf{u} \approx \mathbf{v}$ is deducible from $\Sigma$ if any semigroup that satisfies the identities in $\Sigma$ also satisfies $\mathbf{u} \approx \mathbf{v}$; this is indicated by $\Sigma \vdash \mathbf{u} \approx \mathbf{v}$ or $\mathbf{u} \stackrel{\Sigma}{\approx} \mathbf{v}$. The variety defined by $\Sigma$ is the class of all semigroups that satisfy all identities in $\Sigma$; in this case, $\Sigma$ is a basis for the variety. A variety is finitely based if it possesses a finite basis. The subvariety of a variety $\mathrm{V}$ defined by $\Sigma$ is denoted by $\mathrm{V} \Sigma$.

An identity $\sigma$ deletes to an identity $\sigma^{\prime}$ if, up to renaming of letters, $\sigma^{\prime}$ is obtained from $\sigma$ by removing all occurrences of some letters in $\sigma$. For example, the identity $x y x y z x \approx z x^{2} y$ deletes to the identities $a^{2} x_{7} a \approx x_{7} a^{2}$ and $p^{2} y_{2} \approx y_{2} p$.

For any varieties $\mathrm{U}$ and $\mathrm{V}$ such that $\mathrm{U} \subseteq \mathrm{V}$, the interval $[\mathrm{U}, \mathrm{V}]$ is the set of all subvarieties of $\mathrm{V}$ containing $\mathrm{U}$. The lattice of subvarieties of $\mathrm{V}$ is denoted by $\mathcal{L}(\mathrm{V})$. Note that $\mathcal{L}(\mathrm{V})=[\mathbf{0}, \mathrm{V}]$ where $\mathbf{0}$ is the variety of trivial semigroups. 


\subsection{Some 2-testable monoids}

It is routine to check that the subsets

$$
A_{0}=\{0, b, a b, b a\}, \quad L_{2}=\{a, b a\}, \quad R_{2}=\{a, a b\}, \quad \text { and } \quad N_{2}=\{0, b\}
$$

of $A_{2}$ are subsemigroups of $A_{2}$, and that the subset

$$
B_{0}=\{0, d, c d, d c\}
$$

of $B_{2}$ is a subsemigroup of $B_{2}$. Note that $L_{2}$ is a left-zero semigroup, $R_{2}$ is a right-zero semigroup, and $N_{2}$ is a null semigroup. The variety $\operatorname{VAR}\left\{B_{0}\right\}$ is the unique maximal subvariety of both the varieties $\operatorname{Var}\left\{A_{0}\right\}$ and $\operatorname{VaR}\left\{B_{2}\right\}[6]$; a description of all subvarieties of $\operatorname{VAR}_{A}\left\{B_{0}\right\}$ is given by Lee [7]. Let $A_{0}^{1}, B_{0}^{1}, L_{2}^{1}, R_{2}^{1}$, and $N_{2}^{1}$ be the varieties generated by the 2-testable monoids $A_{0}^{1}, B_{0}^{1}, L_{2}^{1}, R_{2}^{1}$, and $N_{2}^{1}$, respectively.

An identity $\mathbf{u} \approx \mathbf{v}$ is quadratic if the words $\mathbf{u}$ and $\mathbf{v}$ are quadratic. Define a relation $\stackrel{\circ}{=}$ on $\mathcal{X}^{*}$ by $\mathbf{u} \stackrel{\circ}{=}$ if the words $\mathbf{u}$ and $v$ can be obtained from one another by rearrangement of letters. It is easy to verify the following well-known results.

Lemma 2.1.

Let $\mathbf{u} \approx \mathbf{v}$ be any identity. Then

(i) $\mathrm{L}_{2}^{1} \vDash \mathbf{u} \approx \mathbf{v}$ if and only if ini $(\mathbf{u})=\operatorname{ini}(\mathrm{v})$;

(ii) $\mathbf{R}_{2}^{1} \vDash \mathbf{u} \approx \mathbf{v}$ if and only if fin $(\mathbf{u})=$ fin $(\mathrm{v})$.

Further, if the identity $\mathbf{u} \approx \mathrm{v}$ is quadratic, then

(iii) $\mathrm{N}_{2}^{1} \vDash \mathbf{u} \approx \mathbf{v}$ if and only if $\mathbf{u} \stackrel{\circ}{=} \mathbf{v}$.

Lemma 2.2.

Let $\mathbf{u} \approx \mathbf{v}$ be any quadratic identity such that $\operatorname{con}(\mathbf{u})=\operatorname{con}(\mathbf{v})$. Then

(i) $\mathrm{A}_{0}^{1} \vDash \mathbf{u} \approx \mathrm{v}$ if and only if $\mathbf{u} \approx \mathrm{v}$ does not delete to any of the following identities:

$$
\begin{aligned}
& x^{2} \approx x, \quad x y \approx y x, \quad x y x \approx x^{2} y, \quad x y x \approx y x^{2}, \quad x^{2} y \approx y x^{2}, \\
& x y^{2} x \approx x^{2} y^{2}, \quad x y^{2} x \approx y^{2} x^{2}, \quad x y x y \approx x^{2} y^{2}, \quad x y x y \approx y^{2} x^{2}, \quad x^{2} y^{2} \approx y^{2} x^{2} ;
\end{aligned}
$$

(ii) $\mathrm{B}_{0}^{1} \vDash \mathbf{u} \approx \mathbf{v}$ if and only if $\mathbf{u} \approx \mathbf{v}$ does not delete to any identity in (2).

Proof. Part (i) follows from Edmunds [3, Lemma 4.1 and the proof of Proposition 3.2(a)]. Part (ii) follows from Edmunds [3, proof of Proposition 3.1(i)].

\section{Proposition 2.3.}

(i) The variety $\mathrm{A}_{0}^{1} \vee \mathrm{L}_{2}^{1} \vee \mathrm{R}_{2}^{1}$ is defined by the identities

$$
x y x z x \approx x y z x, \quad x^{2} y x \approx x y x, \quad x y x^{2} \approx x y x, \quad x^{3} \approx x^{2} .
$$

(ii) The variety $\mathrm{A}_{0}^{1} \vee \mathrm{L}_{2}^{1}$ is defined by the identities ( $\star$ ) and

$$
x y x y \approx x y^{2} x
$$


(iii) The variety $A_{0}^{1} \vee R_{2}^{1}$ is defined by the identities ( $\star$ ) and

$$
x y x y \approx y x^{2} y
$$

(iv) The variety $A_{0}^{1}$ is defined by the identities ( $\star$ ), $(\triangleright)$, and ( $\left.\triangleleft\right)$.

\section{Remark 2.4.}

Note that if a letter $x$ occurs three or more times in a word $\mathbf{w}$, then all except the first and last occurrences of $x$ in $\mathbf{w}$ can be eliminated by the identities $(\star)$. Therefore any word can be converted by the identities ( $\star$ ) into a unique quadratic word.

Proof of Proposition 2.3. Parts (ii)-(iv) are consequences of Edmunds [3, Propositions 3.2(a)] and Lee [11, Proposition 3.3 and Corollary 3.4]. Therefore it remains to verify part (i).

It is routine to show that the monoids $A_{0}^{1}, L_{2}^{1}$, and $R_{2}^{1}$ satisfy the identities $(\star)$. Therefore, to complete the proof, it suffices to show that any identity $\mathbf{u} \approx \mathbf{v}$ of the variety $A_{0}^{1} \vee L_{2}^{1} \vee R_{2}^{1}$ is deducible from the identities $(\star)$. Since the variety $A_{0}^{1} \vee L_{2}^{1} \vee R_{2}^{1}$ satisfies the identities $(\star)$, it follows from Remark 2.4 that the words $u$ and $v$ can be assumed to be quadratic. Hence $\mathbf{u}$ and $\mathbf{v}$ can be written as $\mathbf{u}=\mathbf{s}_{1} \mathbf{u}_{1} \cdots \mathbf{s}_{m} \mathbf{u}_{m}$ and $\mathbf{v}=\mathbf{t}_{1} \mathbf{v}_{1} \cdots \mathbf{t}_{n} \mathbf{v}_{n}$, where

- the letters of $\mathbf{s}_{1} \in \mathcal{X}^{*}$ and $\mathbf{s}_{2}, \ldots, \mathbf{s}_{m} \in \mathcal{X}^{+}$are all simple in $\mathbf{u}$,

- the letters of $\mathbf{u}_{1}, \ldots, \mathbf{u}_{m-1} \in \mathcal{X}^{+}$and $\mathbf{u}_{m} \in \mathcal{X}^{*}$ are all non-simple in $\mathbf{u}$,

- the letters of $\mathbf{t}_{1} \in \mathcal{X}^{*}$ and $\mathbf{t}_{2}, \ldots, \mathbf{t}_{n} \in \mathcal{X}^{+}$are all simple in $\mathbf{v}$,

- the letters of $\mathbf{v}_{1}, \ldots, \mathbf{v}_{n-1} \in \mathcal{X}^{+}$and $\mathbf{v}_{n} \in \mathcal{X}^{*}$ are all non-simple in $\mathbf{v}$.

Since the identity $\mathbf{u} \approx \mathbf{v}$ is satisfied by the monoids $A_{0}^{1}, L_{2}^{1}$, and $R_{2}^{1}$, it follows from Lemmas 2.1 and $2.2(\mathrm{i})$ that

(a) ini $(\mathbf{u})=\operatorname{ini}(\mathbf{v})$ and fin $(\mathbf{u})=$ fin $(\mathbf{v})$,

(b) $\mathbf{u} \approx \mathbf{v}$ does not delete to any identity from $\{(2),(3)\}$;

specifically,

(c) $\mathbf{u} \approx \mathrm{v}$ does not delete to the identity $x y x y \approx x^{2} y^{2}$.

Since $\mathbf{u}$ and $\mathbf{v}$ are quadratic words, it follows from (b) that $m=n, \mathbf{s}_{i}=\mathbf{t}_{i}$, and $\mathbf{u}_{i} \stackrel{\circ}{=} \mathbf{v}_{i}$ for all $i$. Therefore $\mathbf{v}=\mathbf{s}_{1} \mathbf{v}_{1} \cdots \mathbf{s}_{m} \mathbf{v}_{m}$. It then follows from (a) and (c) that $\mathbf{u}_{i}=\mathbf{v}_{i}$ for all $i$, whence the identity $\mathbf{u} \approx \mathbf{v}$ is trivial. Consequently, the identity $\mathbf{u} \approx \mathbf{v}$ is deducible from the identities $(\star)$.

\section{Proof of Theorem 1.2(ii)}

It is routine to show that the semigroup $C_{0}$ with the following multiplication table satisfies the identities (1) and so belongs to the variety $A_{2}$ :

\begin{tabular}{c|cccccc}
$c_{0}$ & 0 & $a$ & $b$ & $c$ & $d$ & $e$ \\
\hline 0 & 0 & 0 & 0 & 0 & 0 & 0 \\
$a$ & 0 & 0 & 0 & 0 & $a$ & $a$ \\
$b$ & 0 & $a$ & $b$ & $c$ & 0 & $a$ \\
$c$ & 0 & $a$ & $b$ & $c$ & $a$ & $a$ \\
$d$ & 0 & 0 & 0 & 0 & $d$ & $d$ \\
$e$ & 0 & 0 & 0 & 0 & $e$ & $e$
\end{tabular}


The semigroups $A_{0}, B_{0}, L_{2}, R_{2}$, and $N_{2}$ are isomorphic to the subsemigroups

$$
\{0, a, b, e\}, \quad\{0, a, b, d\}, \quad\{b, c\}, \quad\{d, e\}, \quad \text { and } \quad\{0, a\}
$$

of $C_{0}$ respectively. Let $\mathrm{A}_{0}=\operatorname{VaR}\left\{A_{0}\right\}, \mathrm{B}_{0}=\operatorname{VAR}\left\{B_{0}\right\}, \mathrm{B}_{2}=\operatorname{VaR}\left\{B_{2}\right\}, \mathrm{C}_{0}=\operatorname{VaR}\left\{C_{0}\right\}$, and $C_{0}^{1}=\operatorname{VaR}\left\{C_{0}^{1}\right\}$.

\section{Lemma 3.1.}

The variety $\mathrm{C}_{0}$ is the subvariety of $\mathrm{A}_{2}$ that is largest with respect to not containing the semigroup $B_{2}$.

Proof. This follows from Lee [6, Theorem 3.6] and Lee and Volkov [15, Theorem 4.2(iii)].

A complete description of the interval $\left[B_{0}, A_{2}\right]$ can be found in Lee [12, Figure 5]. The varieties $C_{0}$ (denoted in Lee [12] by $\bar{B}_{2}$ ) and $A_{2}$ are very close since the interval $\left[C_{0}, A_{2}\right]$ coincides with the chain $C_{0} \subset B_{2} \vee C_{0} \subset A_{2}$.

\section{Lemma 3.2.}

$$
C_{0}^{1}=A_{0}^{1} \vee L_{2}^{1} \vee R_{2}^{1}
$$

Proof. Since the monoids $A_{0}^{1}, L_{2}^{1}$, and $R_{2}^{1}$ are embeddable in $C_{0}^{1}$, the inclusion $A_{0}^{1} \vee L_{2}^{1} \vee R_{2}^{1} \subseteq C_{0}^{1}$ holds. It is routine to show that the monoid $C_{0}^{1}$ satisfies the identities $(\star)$ so that the inclusion $C_{0}^{1} \subseteq A_{0}^{1} \vee L_{2}^{1} \vee R_{2}^{1}$ holds by Proposition 2.3(i).

\section{Theorem 3.3.}

The variety $\mathrm{C}_{0}^{1}$ is hereditarily finitely based. Equivalently, any semigroup that satisfies the identities ( $\star$ ) is finitely based.

Theorem 3.3 will be established over the next three sections. Restrictions on identities that can be used to define varieties in the interval $\left[\mathrm{A}_{0}, \mathrm{C}_{0}^{1}\right]$ are given in Section 4. These restrictions are then used in Sections 5 and 6 to show that all subvarieties of $C_{0}^{1}$ are finitely based. Specifically, all subvarieties of $C_{0}^{1}$ that contain the variety $A_{0} \vee B_{0}^{1}$ are shown to be finitely based in Proposition 5.1, while those that do not contain the variety $A_{0} \vee B_{0}^{1}$ are shown to be finitely based in Proposition 6.1.

Let $S^{1}$ be any 2-testable monoid such that $B_{2} \notin V_{A R}\{S\}$. By Lemma 3.1, the semigroup $S$ belongs to the variety $C_{0}$ so that the monoid $S^{1}$ belongs to the variety $C_{0}^{1}$. By Theorem 3.3, the monoid $S^{1}$ is hereditarily finitely based.

\section{Identities of varieties containing $A_{0}$}

Recall from Proposition 2.3(i) and Lemma 3.2 that the variety $C_{0}^{1}$ is defined by the identities ( $\star$ ). Since most equational deductions in the remainder of this article are deductions within the equational theory of the semigroup $C_{0}^{1}$, it is convenient to write $\Sigma_{1} \Vdash \Sigma_{2}$, where $\Sigma_{1}$ and $\Sigma_{2}$ are any sets of identities, to stand for the deduction $(\star) \cup \Sigma_{1} \vdash \Sigma_{2}$.

Two words are said to be disjoint if they do not share any common letter. A word of length at least two is said to be connected if it cannot be written as a product of two disjoint nonempty words. An identity $\mathbf{u} \approx \mathbf{v}$ is connected if the words $\mathbf{u}$ and $\mathbf{v}$ are connected.

\section{Lemma 4.1.}

Let $\mathbf{u}$ and $\mathbf{v}$ be any words.

(i) Suppose that $\mathbf{u}=\mathbf{u}_{1} \cdots \mathbf{u}_{m}$ (respectively, $\mathbf{v}=\mathbf{v}_{1} \cdots \mathbf{v}_{n}$ ) where $\mathbf{u}_{1}, \ldots, \mathbf{u}_{m}$ (respectively, $\mathbf{v}_{1}, \ldots, \mathbf{v}_{n}$ ) are pairwise disjoint words each of which is either connected or a singleton. Then $\mathrm{A}_{0} \vDash \mathbf{u} \approx \mathbf{v}$ if and only if $m=n$ and $\mathrm{A}_{0} \vDash \mathbf{u}_{i} \approx \mathrm{v}_{i}$ for all $i$. 
(ii) Suppose that either $\mathbf{u}$ or $\mathbf{v}$ is a simple word. Then $\mathrm{A}_{\mathbf{0}} \vDash \mathbf{u} \approx \mathbf{v}$ if and only if $\mathbf{u}=\mathbf{v}$.

(iii) Suppose that $\mathbf{u}$ and $\mathbf{v}$ are connected words. Then $\mathrm{A}_{0} \vDash \mathbf{u} \approx \mathbf{v}$ if and only if con $(\mathrm{u})=\operatorname{con}(\mathrm{v})$.

Proof. These results follow from the proof of part 4 of the first proposition in Edmunds [4].

For any $\ell$ and $r$ from the set

$$
\mathbb{N}^{\star}=\{0,1,2, \ldots, \infty, \infty+1\}
$$

define the words

$$
\mathbf{p}_{(\ell)}=\left\{\begin{array}{ll}
\emptyset & \text { if } \ell=0, \\
p_{1} \cdots p_{\ell} & \text { if } 1 \leq \ell<\infty, \\
p_{1}^{2} p_{2} & \text { if } \ell=\infty, \\
p_{1}^{2} & \text { if } \ell=\infty+1,
\end{array} \quad \text { and } \quad \mathbf{q}_{(r)}= \begin{cases}\emptyset & \text { if } r=0, \\
q_{1} \cdots q_{r} & \text { if } 1 \leq r<\infty, \\
q_{1} q_{2}^{2} & \text { if } r=\infty, \\
q_{2}^{2} & \text { if } r=\infty+1\end{cases}\right.
$$

\section{Lemma 4.2.}

Each variety in the interval $\left[\mathrm{A}_{0}, \mathrm{C}_{0}^{1}\right]$ is defined within the variety $\mathrm{C}_{0}^{1}$ by quadratic identities of the form

$$
\mathbf{p}_{(\ell)} \mathbf{u} \mathbf{q}_{(r)} \approx \mathbf{p}_{(\ell)} \mathbf{v} \mathbf{q}_{(r)}
$$

where

(1†) $\ell, r \in \mathbb{N}^{\star}$

$(2 \dagger) \mathrm{u}, \mathrm{v} \in \mathcal{X}^{+}$are connected and quadratic;

(3†) $\mathbf{p}_{(\ell)}, \mathbf{u}, \mathbf{q}_{(r)}$ are pairwise disjoint and $\mathbf{p}_{(\ell)}, \mathbf{v}, \mathbf{q}_{(r)}$ are pairwise disjoint;

(4†) $\mathrm{A}_{0} \vDash \mathbf{u} \approx \mathrm{v}$ so that $\operatorname{con}(\mathrm{u})=\operatorname{con}(\mathrm{v})$;

(5十) $\ell=0$ if $\mathrm{h}(\mathbf{u})=\mathrm{h}(\mathbf{v})$;

(6†) $r=0$ if $\mathrm{t}(\mathbf{u})=\mathrm{t}(\mathbf{v})$.

Proof. It suffices to consider a variety in the interval $\left[A_{0}, C_{0}^{1}\right]$ of the form $C_{0}^{1}\{\xi\}$, where

$$
\xi: \mathbf{a} \approx \mathbf{b}
$$

is any nontrivial, quadratic identity of the variety $A_{0}$, and show that

$$
C_{0}^{1}\{\xi\}=C_{0}^{1}\left\{\xi_{1}, \ldots, \xi_{k}\right\}
$$

for some identities $\xi_{1}, \ldots, \xi_{k}$ of the form (4) that satisfy conditions (1†)-(6†). By Lemma 4.1(ii), the words a and $\mathbf{b}$ are non-simple. By Remark 2.4 and Lemma 4.1(i),

$$
\mathbf{a}=\mathbf{s}_{1} \mathbf{u}_{1} \mathbf{s}_{2} \mathbf{u}_{2} \cdots \mathbf{s}_{k} \mathbf{u}_{k} \mathbf{s}_{k+1} \quad \text { and } \quad \mathbf{b}=\mathbf{s}_{1} \mathbf{v}_{1} \mathbf{s}_{2} \mathbf{v}_{2} \cdots \mathbf{s}_{k} \mathbf{v}_{k} \mathbf{s}_{k+1}
$$

where

- $\mathbf{s}_{1}, \ldots, \mathbf{s}_{k+1} \in \mathcal{X}^{*}$ are simple;

- $\mathbf{u}_{1}, \ldots, \mathbf{u}_{k}, \mathbf{v}_{1}, \ldots, \mathbf{v}_{k} \in \mathcal{X}^{+}$are quadratic and connected; 
- $\mathbf{s}_{1}, \mathbf{u}_{1}, \ldots, \mathbf{s}_{k}, \mathbf{u}_{k}, \mathbf{s}_{k+1}$ are pairwise disjoint and $\mathbf{s}_{1}, \mathbf{v}_{1}, \ldots, \mathbf{s}_{k}, \mathbf{v}_{k}, \mathbf{s}_{k+1}$ are pairwise disjoint;

- $\mathrm{A}_{0} \vDash \mathrm{u}_{i} \approx \mathrm{v}_{i}$ (so that $\operatorname{con}\left(\mathrm{u}_{i}\right)=$ con $\left.\left(\mathrm{v}_{i}\right)\right)$ for all $i \in\{1, \ldots, k\}$.

Without loss of generality, assume that $\operatorname{con}\left(\mathbf{p}_{(\ell)} \mathbf{q}_{(r)}\right) \cap \operatorname{con}(\mathbf{a b})=\emptyset$ for all $\ell, r \in \mathbb{N}^{\star}$.

First consider the case when $k=1$. Then the identity $\xi$ is $\mathbf{s}_{1} \mathbf{u}_{1} \mathbf{s}_{2} \approx \mathbf{s}_{1} \mathbf{v}_{1} \mathbf{s}_{2}$, so that the equation (5) holds with the identity $\xi_{1}$ being $\mathbf{p}_{(\ell)} \mathbf{u}_{1} \mathbf{q}_{(r)} \approx \mathbf{p}_{(\ell)} \mathbf{v}_{1} \mathbf{q}_{(r)}$, where $\ell=\left|\mathbf{s}_{1}\right|$ and $r=\left|\mathbf{s}_{2}\right|$.

Now suppose that $k \geq 2$. Denote by $\gamma_{1}$ the substitution

$$
x \mapsto \begin{cases}q_{1} & \text { if } x=\mathrm{h}\left(\mathbf{s}_{2}\right) \\ q_{2}^{2} & \text { if } x \text { occurs after } \mathrm{h}\left(\mathrm{s}_{2}\right) \text { in a. }\end{cases}
$$

Then

$$
\xi \Vdash \mathbf{s}_{1} \mathbf{u}_{1} q_{1} q_{2}^{2} \stackrel{(\star)}{\approx} \mathbf{a} \gamma_{1} \stackrel{\xi}{\approx} \mathbf{b} \gamma_{1} \stackrel{(\star)}{\approx} \mathbf{s}_{1} \mathbf{v}_{1} q_{1} q_{2}^{2} \vdash \xi_{1}: \mathbf{s}_{1} \mathbf{u}_{1} q_{1} q_{2}^{2} \approx \mathbf{s}_{1} \mathbf{v}_{1} q_{1} q_{2}^{2}
$$

where $q_{1}=\varnothing$ if and only if $\mathbf{s}_{2}=\varnothing$, whence the identity $\xi_{1}$ is equivalent to $\mathbf{p}_{(\ell)} \mathbf{u}_{1} \mathbf{q}_{(r)} \approx \mathbf{p}_{(\ell)} \mathbf{v}_{1} \mathbf{q}_{(r)}$ with $\ell=\left|\mathbf{s}_{1}\right|$ and $r \in\{\infty, \infty+1\}$. For $1<i<k$, denote by $\gamma_{i}$ the substitution

$$
x \mapsto \begin{cases}p_{1}^{2} & \text { if } x \text { occurs before } \mathrm{t}\left(\mathbf{s}_{i}\right) \text { in } \mathbf{a}, \\ p_{2} & \text { if } x=\mathrm{t}\left(\mathbf{s}_{i}\right), \\ q_{1} & \text { if } x=\mathrm{h}\left(\mathbf{s}_{i+1}\right), \\ q_{2}^{2} & \text { if } x \text { occurs after } \mathrm{h}\left(\mathbf{s}_{i+1}\right) \text { in } \mathbf{a} .\end{cases}
$$

Then

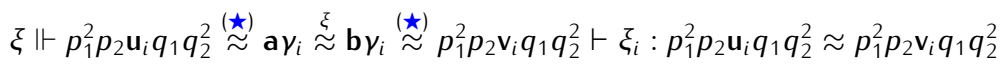

where $p_{2}=\varnothing$ if and only if $\mathbf{s}_{i}=\varnothing$, and $q_{1}=\varnothing$ if and only if $\mathbf{s}_{i+1}=\varnothing$, whence the identity $\xi_{i}$ is equivalent to $\mathbf{p}_{(\ell)} \mathbf{u}_{i} \mathbf{q}_{(r)} \approx \mathbf{p}_{(\ell)} \mathbf{v}_{i} \mathbf{q}_{(r)}$ with $\ell, r \in\{\infty, \infty+1\}$. Finally, denote by $\gamma_{k}$ the substitution

$$
x \mapsto \begin{cases}p_{1}^{2} & \text { if } x \text { occurs before } \mathrm{t}\left(\mathbf{s}_{k}\right) \text { in } \mathbf{a}, \\ p_{2} & \text { if } x=\mathrm{t}\left(\mathbf{s}_{k}\right) .\end{cases}
$$

Then

$$
\xi \Vdash p_{1}^{2} p_{2} \mathbf{u}_{k} \mathbf{s}_{k+1} \stackrel{(\star)}{\approx} \mathbf{a} \gamma_{k} \stackrel{\xi}{\approx} \mathbf{b} \gamma_{k} \stackrel{(\star)}{\approx} p_{1}^{2} p_{2} \mathbf{v}_{k} \mathbf{s}_{k+1} \vdash \xi_{k}: p_{1}^{2} p_{2} \mathbf{u}_{k} \mathbf{s}_{k+1} \approx p_{1}^{2} p_{2} \mathbf{v}_{k} \mathbf{s}_{k+1}
$$

where $p_{2}=\emptyset$ if and only if $\mathbf{s}_{k}=\emptyset$, whence the identity $\xi_{k}$ is equivalent to $\mathbf{p}_{(\ell)} \mathbf{u}_{k} \mathbf{q}_{(r)} \approx \mathbf{p}_{(\ell)} \mathbf{v}_{k} \mathbf{q}_{(r)}$ with $\ell \in\{\infty, \infty+1\}$ and $r=\left|\mathbf{s}_{k+1}\right|$. Hence the deduction $\xi \Vdash\left\{\xi_{1}, \ldots, \xi_{k}\right\}$ holds and the inclusion $\mathbf{C}_{0}^{1}\{\xi\} \subseteq \mathrm{C}_{0}^{1}\left\{\xi_{1}, \ldots, \xi_{k}\right\}$ is established.

It remains to verify the deduction $\left\{\xi_{1}, \ldots, \xi_{k}\right\} \Vdash \xi$ so that the equation (5) holds. For each $i$, the word $\mathbf{u}_{i}$ is connected so that the letter $h_{i}=\mathrm{h}\left(\mathbf{u}_{i}\right)$ occurs at least twice in $\mathbf{u}_{i}$. Therefore $\mathbf{u}_{i} \stackrel{(\star)}{\approx} h_{i}^{2} \mathbf{u}_{i}$. Similarly, the letter $t_{i}=\mathrm{t}\left(\mathbf{v}_{i}\right)$ occurs at least twice in the word $v_{i}$ so that $v_{i} \stackrel{(\star)}{\approx} v_{i} t_{i}^{2}$. The deduction $\left\{\xi_{1}, \ldots, \xi_{k}\right\} \Vdash \xi$ then follows since

$$
\begin{aligned}
& \mathbf{a}=\mathbf{s}_{1} \mathbf{u}_{1} \mathbf{s}_{2} \mathbf{u}_{2} \mathbf{s}_{3} \mathbf{u}_{3} \cdots \mathbf{s}_{k} \mathbf{u}_{k} \mathbf{s}_{k+1} \stackrel{(\star)}{\approx}\left(\mathbf{s}_{1} \mathbf{u}_{1} \mathbf{s}_{2} h_{2}^{2}\right) \mathbf{u}_{2} \mathbf{s}_{3} \mathbf{u}_{3} \cdots \mathbf{s}_{k} \mathbf{u}_{k} \mathbf{s}_{k+1} \\
& \stackrel{\xi_{1}}{\approx} \mathbf{s}_{1} \mathbf{v}_{1} \mathbf{s}_{2}\left(h_{2}^{2} \mathbf{u}_{2}\right) \mathbf{s}_{3} \mathbf{u}_{3} \cdots \mathbf{s}_{k} \mathbf{u}_{k} \mathbf{s}_{k+1} \stackrel{(\star)}{\approx} \mathbf{s}_{1} \mathbf{v}_{1}\left(t_{1}^{2} \mathbf{s}_{2} \mathbf{u}_{2} \mathbf{s}_{3} h_{3}^{2}\right) \mathbf{u}_{3} \cdots \mathbf{s}_{k} \mathbf{u}_{k} \mathbf{s}_{k+1} \\
& \stackrel{\xi_{2}}{\approx} \mathbf{s}_{1}\left(\mathbf{v}_{1} t_{1}^{2}\right) \mathbf{s}_{2} \mathbf{v}_{2} \mathbf{s}_{3}\left(h_{3}^{2} \mathbf{u}_{3}\right) \cdots \mathbf{s}_{k} \mathbf{u}_{k} \mathbf{s}_{k+1} \stackrel{(\star)}{\approx} \mathbf{s}_{1} \mathbf{v}_{1} \mathbf{s}_{2} \mathbf{v}_{2}\left(t_{2}^{2} \mathbf{s}_{3} \mathbf{u}_{3} \mathbf{s}_{4} h_{4}^{2}\right) \mathbf{u}_{4} \cdots \mathbf{s}_{k} \mathbf{u}_{k} \mathbf{s}_{k+1} \\
& \stackrel{\xi_{3}}{\approx} \ldots \stackrel{(\star)}{\approx} \ldots \stackrel{\xi_{4}}{\approx} \ldots \stackrel{(\star)}{\approx} \mathbf{s}_{1} \mathbf{v}_{1} \mathbf{s}_{2} \mathbf{v}_{2} \ldots \mathbf{s}_{k-1} \mathbf{v}_{k-1}\left(t_{k-1}^{2} \mathbf{s}_{k} \mathbf{u}_{k} \mathbf{s}_{k+1}\right) \\
& \stackrel{\xi_{k}}{\approx} \mathbf{s}_{1} \mathbf{v}_{1} \mathbf{s}_{2} \mathbf{v}_{2} \cdots \mathbf{s}_{k-1}\left(\mathbf{v}_{k-1} t_{k-1}^{2}\right) \mathbf{s}_{k} \mathbf{v}_{k} \mathbf{s}_{k+1} \stackrel{(\star)}{\approx} \mathbf{s}_{1} \mathbf{v}_{1} \mathbf{s}_{2} \mathbf{v}_{2} \cdots \mathbf{s}_{k-1} \mathbf{v}_{k-1} \mathbf{s}_{k} \mathbf{v}_{k} \mathbf{s}_{k+1}=\mathbf{b} .
\end{aligned}
$$


Now each identity $\xi_{i}$ is of the form (4) and it is easy to see that it satisfies conditions (1†)-(4†). Suppose that $\mathrm{h}(\mathbf{u})=\mathrm{h}(\mathbf{v})=h$. Since $\mathbf{u}$ and $\mathbf{v}$ are connected words, the letter $h$ occurs more than once in both $\mathbf{u}$ and $\mathbf{v}$. Therefore

$$
\xi_{i} \Vdash \mathbf{u q}_{(r)} \stackrel{(\star)}{\approx} h^{|\mathbf{p}(\ell)|} \mathbf{u q}_{(r)} \stackrel{\xi_{i}}{\approx} h^{|\mathbf{p}(\ell)|} \mathbf{v q}_{(r)} \stackrel{(\star)}{\approx} \mathbf{v} \mathbf{q}_{(r)} \vdash \mathbf{u} \mathbf{q}_{(r)} \approx \mathbf{v} \mathbf{q}_{(r)},
$$

whence the identity $\xi_{i}$ can be chosen to satisfy condition (5†). By a symmetrical argument, each identity $\xi_{i}$ can also be chosen to satisfy condition (6†).

\section{Proof of Theorem 3.3: subvarieties containing $A_{0} \vee B_{0}^{1}$}

\section{Proposition 5.1.}

Any variety in the interval $\left[\mathrm{A}_{0} \vee \mathrm{B}_{0}^{1}, \mathrm{C}_{0}^{1}\right]$ is finitely based.

Proof. Let $\mathrm{V}$ be any variety in the interval $\left[\mathrm{A}_{0} \vee \mathrm{B}_{0}^{1}, \mathrm{C}_{0}^{1}\right]$.

Case 1: $A_{0}^{1} \subseteq \mathrm{V}$. Then $\mathrm{V} \in\left[\mathrm{A}_{0}^{1}, \mathrm{C}_{0}^{1}\right]$ and $\mathrm{V}$ is shown to be finitely based in Subsection 5.1.

Case 2: $A_{0}^{1} \nsubseteq \mathbb{V}$. Then $V$ is shown to be finitely based in Subsection 5.2.

\subsection{Varieties in $\left[A_{0}^{1}, C_{0}^{1}\right]$}

The main result of this subsection, Proposition 5.8, establishes the finite basis property of every variety in the interval $\left[\mathrm{A}_{0}^{1}, \mathrm{C}_{0}^{1}\right]$.

\section{Lemma 5.2.}

Let $\mathrm{V}$ be any subvariety of $\mathrm{C}_{0}^{1}$.

(i) If $R_{2}^{1} \notin \mathrm{V}$, then the variety $\mathrm{V}$ satisfies the identity

$$
h x y x y h \approx h x y^{2} x h
$$

(ii) If $L_{2}^{1} \notin \mathrm{V}$, then the variety $\mathrm{V}$ satisfies the identity

$$
h x y x y h \approx h y x^{2} y h
$$

Proof. (i) It follows from Almeida [1, Proposition 10.10.2(b)] that the identity $x y x y \approx x y^{2} x$ defines the subvariety of $\mathrm{C}_{0}^{1}$ that is largest with respect to not containing $R_{2}$. Suppose that $R_{2}^{1} \notin \mathrm{V}$. Let $\varphi$ be the substitution $z \mapsto h^{2} z h^{2}$ for all $z \in \mathcal{X}$. Then it follows from Lee [8, Theorem 2] that the variety $\mathrm{V}$ satisfies the identity $(x y x y) \varphi \approx\left(x y^{2} x\right) \varphi$. It is easy to deduce that the variety $\mathrm{V}$ also satisfies the identity (6).

(ii) This is symmetrical to part (i).

The identities

$$
\lambda_{m}: \mathbf{p}_{(m)} x y x y \approx \mathbf{p}_{(m)} y x^{2} y, \quad \rho_{m}: x y x y \mathbf{q}_{(m)} \approx x y^{2} x \mathbf{q}_{(m)},
$$

where $m \in \mathbb{N}^{\star}$, are required in the present subsection. It is straightforward to verify the following lemma. 


\section{Lemma 5.3.}

The following deductions hold:

(i) $\lambda_{0} \Vdash \lambda_{1} \Vdash \cdots \Vdash \lambda_{\infty} \Vdash \lambda_{\infty+1} \Vdash(7)$;

(ii) $\rho_{0} \Vdash \rho_{1} \Vdash \cdots \Vdash \rho_{\infty} \Vdash \rho_{\infty+1} \Vdash(6)$.

\section{Lemma 5.4.}

Let $\mathbf{u} \approx \mathbf{v}$ be any quadratic, connected identity of the variety $\mathrm{A}_{0}^{1}$.

(i) If ini $(\mathbf{u})=\operatorname{ini}(\mathbf{v})$ and $\mathrm{t}(\mathrm{u})=\mathrm{t}(\mathbf{v})$, then $(6) \Vdash \mathbf{u} \approx \mathbf{v}$.

(ii) If $\mathrm{h}(\mathbf{u})=\mathrm{h}(\mathbf{v})$ and fin $(\mathbf{u})=$ fin $(\mathbf{v})$, then (7) $\Vdash \mathbf{u} \approx \mathbf{v}$.

(iii) If $\mathrm{h}(\mathbf{u})=\mathrm{h}(\mathbf{v})$ and $\mathrm{t}(\mathbf{u})=\mathrm{t}(\mathbf{v})$, then $\{(6),(7)\} \Vdash \mathbf{u} \approx \mathrm{v}$.

Proof. (i) Suppose that ini $(\mathbf{u})=\operatorname{ini}(\mathbf{v})$ and $\mathrm{t}(\mathbf{u})=\mathrm{t}(\mathbf{v})$. Then by Lemma 2.1(i), the identity $\mathbf{u} \approx \mathbf{v}$ is satisfied by the variety $A_{0}^{1} \vee L_{2}^{1}$. Recall from Proposition 2.3(ii) that the variety $A_{0}^{1} \vee L_{2}^{1}$ is defined by the identities $\{(\star)$, ( $\left.\triangleright)\right\}$. It follows that there exists a deduction sequence

$$
\mathbf{u}=\mathbf{w}_{0} \Rightarrow \mathbf{w}_{1} \Rightarrow \ldots \Rightarrow \mathbf{w}_{r}=\mathbf{v}
$$

where each deduction $\mathbf{w}_{j} \Rightarrow \mathbf{w}_{j+1}$ involves an identity from $\{(\star),(\nabla)\}$. (Recall that a deduction $\mathbf{w} \Rightarrow \mathbf{w}^{\prime}$ involves an identity $\mathbf{z} \approx \mathbf{z}^{\prime}$ if there exist words $\mathbf{h}, \mathbf{t} \in \mathcal{X}^{*}$ and an endomorphism $\varphi$ of $\mathcal{X}^{+}$such that $\mathbf{w}=\mathbf{h}(\mathbf{z} \varphi) \mathbf{t}$ and $\mathbf{w}^{\prime}=\mathbf{h}\left(\mathbf{z}^{\prime} \varphi\right) \mathbf{t}$.) For each $j$, since the identity $\mathbf{u} \approx \mathbf{w}_{j}$ is satisfied by the variety $A_{0}$ and the word $\mathbf{u}$ is connected, it follows from Lemma 4.1 that $\mathbf{w}_{j}$ is a connected word with $\operatorname{con}\left(\mathbf{w}_{j}\right)=\operatorname{con}(\mathbf{u})$. Let $t=\mathrm{t}(\mathbf{u})=\mathrm{t}(\mathbf{v})$. Since the deductions $\mathbf{u} \stackrel{(\star)}{\approx} \mathbf{u} t$ and $\mathbf{v} \stackrel{(\star)}{\approx} \mathbf{v} t$ follow from the connectedness of the words $\mathbf{u}$ and $\mathbf{v}$, multiplying every word in the sequence (8a) on the right by the letter $t$ results in the deduction sequence

$$
\mathbf{u} \Rightarrow \mathbf{w}_{0} t \Rightarrow \mathbf{w}_{1} t \Rightarrow \ldots \mathbf{w}_{r} t \Rightarrow \mathbf{v}
$$

(where the first and last deductions involve identities from $(\star)$ ). Hence there is no loss in generality to assume that every word in the sequence (8a) ends with the letter $t$.

Suppose that the deduction $\mathbf{w}_{j} \Rightarrow \mathbf{w}_{j+1}$ in (8a) involves the identity $(\boldsymbol{\nabla})$. Then $\mathbf{w}_{j}=$ abxyc and $\mathbf{w}_{j+1}=$ abyxc for some $\mathbf{x}, \mathbf{y} \in \mathcal{X}^{+}, \mathbf{a}, \mathbf{c} \in \mathcal{X}^{*}$, and $\mathbf{b} \in\{\mathbf{x y}, \mathbf{y x}\}$.

Case 1: $\mathbf{c}=\emptyset$. Then $t=\mathrm{t}(\mathbf{x})=\mathrm{t}(\mathbf{y})$ by assumption. Since $\mathbf{b} \in\{\mathbf{x y}, \mathbf{y x}\}$, the letter $t$ also occurs in $\mathbf{b}$. Therefore

$$
\mathrm{w}_{j}=\mathrm{abxy} \stackrel{(\star)}{\approx} \mathrm{abtyx} \mathrm{x}^{2} \mathrm{y} \stackrel{((6)}{\approx} \mathrm{ab} t \mathrm{yxyx} t \stackrel{(\star)}{\approx} \mathrm{abyx}=\mathrm{w}_{j+1}
$$

Case 2: $c \neq \varnothing$. Since the word $\mathbf{w}_{j}$ is connected, there exists a letter $z$ that is common to both its factors abxy and $\mathbf{c}$. Since $\mathbf{b} \in\{\mathbf{x y}, \mathbf{y} \mathbf{x}\}$, the letter $z$ is common to $\mathbf{a b}$ and $\mathbf{c}$. Therefore

$$
\mathbf{w}_{j}=\mathrm{abxyc} \stackrel{(\star)}{\approx} \mathrm{abzyx} \mathrm{x}^{2} \mathrm{yzc} \stackrel{(6)}{\approx} \mathrm{abzyxyxzc} \stackrel{(\star)}{\approx} \mathrm{abyxc}=\mathbf{w}_{j+1}
$$

It follows from Cases 1 and 2 that any deduction $\mathbf{w}_{j} \Rightarrow \mathbf{w}_{j+1}$ in (8a) that involves the identity $(\boldsymbol{\nabla})$ can be replaced by a deduction sequence that involves identities from $\{(\star),(6)\}$. Consequently, the deduction $(6) \Vdash \mathbf{u} \approx \mathbf{v}$ holds.

(ii) This is symmetrical to part (i).

(iii) Suppose that $h(\mathbf{u})=h(\mathbf{v})$ and $t(\mathbf{u})=t(\mathbf{v})$. By Proposition 2.3(iv), the variety $A_{0}^{1}$ is defined by the identities $\{(\star),(\triangleright),(\varangle)\}$. Hence there exists a deduction sequence

$$
\mathbf{u}=\mathbf{w}_{0} \Rightarrow \mathbf{w}_{1} \Rightarrow \ldots \Rightarrow \mathbf{w}_{r}=\mathbf{v}
$$


where each deduction $\mathbf{w}_{j} \Rightarrow \mathbf{w}_{j+1}$ involves an identity from $\{(\star),(\boldsymbol{\nabla}),(\boldsymbol{\triangleleft})\}$. For each $j$, since the identity $\mathbf{w}_{j} \approx \mathbf{u}$ is satisfied by the variety $A_{0}$ and the word $\mathbf{u}$ is connected, it follows from Lemma 4.1 that $\mathbf{w}_{j}$ is a connected word with $\operatorname{con}\left(\mathbf{w}_{j}\right)=\operatorname{con}(\mathbf{u})$. Let $h=\mathrm{h}(\mathbf{u})=\mathrm{h}(\mathbf{v})$ and $t=\mathrm{t}(\mathbf{u})=\mathrm{t}(\mathbf{v})$. Since the deductions $\mathbf{u} \stackrel{(\star)}{\approx} h \mathbf{u} t$ and $\mathbf{v} \stackrel{(\star)}{\approx} h \mathbf{v} t$ follow from the connectedness of the words $\mathbf{u}$ and $\mathbf{v}$, multiplying every word in the sequence (8b) on the left by the letter $h$ and on the right by the letter $t$ results in the deduction sequence

$$
\mathbf{u} \Rightarrow h \mathbf{w}_{0} t \Rightarrow h \mathbf{w}_{1} t \Rightarrow \ldots \Rightarrow h \mathbf{w}_{r} t \Rightarrow \mathbf{v}
$$

(where the first and last deductions involve identities from $(\star)$ ). Hence there is no loss in generality to assume that every word in the sequence (8b) begins and ends with the letters $h$ and $t$ respectively.

By arguments in the proof of part (i), any deduction $\mathbf{w}_{j} \Rightarrow \mathbf{w}_{j+1}$ in (8b) that involves the identity $(\nabla)$ can be replaced by a deduction sequence that involves identities from $\{(\star),(6)\}$. By symmetry, any deduction $\mathbf{w}_{j} \Rightarrow \mathbf{w}_{j+1}$ in (8b) that involves the identity $(\varangle)$ can be replaced by a deduction sequence that involves identities from $\{(\star)$, (7)\}. Consequently, the deduction $\{(6),(7)\} \Vdash \mathbf{u} \approx \mathbf{v}$ holds.

\section{Lemma 5.5.}

Let $\mathbf{u} \approx \mathbf{v}$ be any quadratic, connected identity of the variety $A_{0}^{1}$ such that con $(\mathbf{u v}) \cap \operatorname{con}\left(\mathbf{p}_{(\ell)} \mathbf{q}_{(r)}\right)=\emptyset$ for some $\ell, r \in \mathbb{N}^{\star}$.

(i) If ini $(\mathbf{u})=\operatorname{ini}(\mathbf{v})$ and $\mathrm{t}(\mathbf{u}) \neq \mathrm{t}(\mathbf{v})$, then $\mathrm{C}_{0}^{1}\left\{(6), \mathbf{u q}_{(r)} \approx \mathbf{v} \mathbf{q}_{(r)}\right\}=\mathrm{C}_{0}^{1}\left\{\rho_{r}\right\}$.

(ii) If $\mathrm{h}(\mathbf{u}) \neq \mathrm{h}(\mathbf{v})$ and fin $(\mathbf{u})=$ fin $(\mathbf{v})$, then $\mathrm{C}_{0}^{1}\left\{(7), \mathbf{p}_{(\ell)} \mathbf{u} \approx \mathbf{p}_{(\ell)} \mathbf{v}\right\}=\mathrm{C}_{0}^{1}\left\{\lambda_{\ell}\right\}$

Proof. (i) Suppose that ini $(\mathbf{u})=\operatorname{ini}(\mathbf{v})$ and $\mathrm{t}(\mathbf{u})=x \neq y=\mathrm{t}(\mathbf{v})$. Since con $(\mathbf{u})=$ con $(\mathbf{v})$ by Lemma 4.1(iii), it follows from Lemma 2.2(i) that the identity $\mathbf{u} \approx \mathbf{v}$ does not delete to any identity in $\{(2),(3)\}$. Hence

$$
\mathbf{u}=\mathbf{a} x \mathbf{b} y \mathbf{c} x \quad \text { and } \quad \mathbf{v}=\mathbf{d} y \mathbf{e} x \mathbf{f} y
$$

for some $\mathbf{a}, \mathbf{b}, \mathbf{c}, \mathbf{d}, \mathbf{e}, \mathbf{f} \in \mathcal{X}^{*}$ with $y \in \operatorname{con}(\mathbf{a b}) \backslash \operatorname{con}(\mathbf{c})$ and $x \in \operatorname{con}(\mathbf{d e}) \backslash \operatorname{con}(\mathbf{f})$. Let $\varphi$ be the substitution $z \mapsto x$ for all $z \in \operatorname{con}(\mathbf{u}) \backslash\{x, y\}$. Then $x y\left(\left(\mathbf{u q}_{(r)}\right) \varphi\right) \stackrel{(\star)}{\approx} x y^{2} x \mathbf{q}_{(r)}$ and $x y\left(\left(\mathbf{v} \mathbf{q}_{(r)}\right) \varphi\right) \stackrel{(\star)}{\approx} x y x y \mathbf{q}_{(r)}$ imply the deduction $\mathbf{u q}_{(r)} \approx \mathbf{v q}_{(r)} \Vdash \rho_{r}$. Hence

$$
\mathbf{C}_{0}^{1}\left\{(6), \mathbf{u q}_{(r)} \approx \mathbf{v q}_{(r)}\right\}=\mathbf{C}_{0}^{1}\left\{(6), \mathbf{u q}_{(r)} \approx \mathbf{v} \mathbf{q}_{(r)}, \rho_{r}\right\}
$$

Note that

(a) if some simple letter $z$ of the word $\mathbf{u}$ belongs to the factor $\mathbf{c}$, then the identity $\mathbf{u} \approx \mathbf{v}$ deletes to the identity $y^{2} z \approx \mathbf{w}$ for some $\mathbf{w} \in\left\{z y^{2}, y z y\right\}$

(b) if both occurrences of some non-simple letter $z$ of the word $\mathbf{u}$ belong to the factor $\mathbf{c}$, then the identity $\mathbf{u} \approx \mathbf{v}$ deletes to the identity $y^{2} z^{2} \approx \mathbf{w}$ for some $\mathbf{w} \in\left\{z^{2} y^{2}, z y z y, y z^{2} y\right\}$.

In both $(\mathrm{a})$ and $(\mathrm{b})$, the identity $\mathbf{u} \approx \mathbf{v}$ deletes to some identity from $\{(2),(3)\}$, contradicting an earlier observation. Thus neither (a) nor (b) is possible, whence each letter in the factor $\mathrm{c}$ is the last occurrence of some non-simple letter of the quadratic word $\mathbf{u}$, that is, each letter in c has a first occurrence somewhere in $\mathbf{a}$ or $\mathbf{b}$. Therefore

$$
\mathbf{u q}_{(r)} \stackrel{(\star)}{\approx} \mathbf{a} x \mathbf{b}\left(x \mathbf{c} y^{2} \mathbf{c} x\right) \mathbf{q}_{(r)} \stackrel{(6)}{\approx} \mathbf{a} x \mathbf{b} x \mathbf{c} y \mathbf{c} y x \mathbf{q}_{(r)} \stackrel{(\star)}{\approx} \mathbf{a} x \mathbf{b c}\left(x y^{2} x \mathbf{q}_{(r)}\right) \stackrel{\rho_{r}}{\approx} \mathbf{a} x \mathbf{b c} x y x y \mathbf{q}_{(r)} \stackrel{(\star)}{\approx} \mathbf{a} x \mathbf{b c} x y \mathbf{q}_{(r)}=\mathbf{u}^{\prime} \mathbf{q}_{(r)},
$$

that is, the deduction $\left\{(6), \rho_{r}\right\} \Vdash \mathbf{u q}_{(r)} \approx \mathbf{u}^{\prime} \mathbf{q}_{(r)}$ holds where $\mathbf{u}^{\prime}=\mathbf{a} x \mathbf{b} \mathbf{c} x y$ is a connected word. Hence

$$
\mathbf{C}_{0}^{1}\left\{(6), \mathbf{u q}_{(r)} \approx \mathbf{v} \mathbf{q}_{(r)}, \rho_{r}\right\}=\mathbf{C}_{0}^{1}\left\{(6), \mathbf{u}^{\prime} \mathbf{q}_{(r)} \approx \mathbf{v} \mathbf{q}_{(r)}, \rho_{r}\right\}
$$

Observe that each identity in $\left\{(\star),(6), \rho_{r}\right\}$ is formed by a pair of words with the same initial part. Therefore, since the identity $\mathbf{u q}_{(r)} \approx \mathbf{u}^{\prime} \mathbf{q}_{(r)}$ is deduced from the identities $\left\{(\star),(6), \rho_{r}\right\}$, it follows that 
(c) $\operatorname{ini}\left(\mathbf{u}^{\prime}\right)=\operatorname{ini}(\mathbf{u})=\operatorname{ini}(\mathbf{v})$.

By Lemma 2.2(i), the variety $A_{0}^{1}$ satisfies the identities $\left\{(\star),(6), \rho_{r}\right\}$ so that

(d) $A_{0}^{1} \vDash u^{\prime} \approx \mathbf{u} \approx \mathbf{v}$.

Now since $\mathrm{t}\left(\mathbf{u}^{\prime}\right)=y=\mathrm{t}(\mathbf{v})$, it follows from (c), (d), and Lemma 5.4(i) that the deduction (6) $\Vdash \mathbf{u}^{\prime} \approx \mathbf{v}$ holds; this deduction, together with the deduction $\rho_{r} \Vdash(6)$ in Lemma 5.3(ii), imply that

$$
\mathbf{C}_{0}^{1}\left\{(6), \mathbf{u}^{\prime} \mathbf{q}_{(r)} \approx \mathbf{v q}_{(r)}, \rho_{r}\right\}=\mathbf{C}_{0}^{1}\left\{\rho_{r}\right\}
$$

The result is now obtained by combining (9a), (9b), and (9c).

(ii) This is symmetrical to part (i).

\section{Lemma 5.6.}

Let $\mathbf{u} \approx \mathbf{v}$ be any quadratic, connected identity of the variety $A_{0}^{1}$ such that con $(\mathbf{u v}) \cap \operatorname{con}\left(\mathbf{p}_{(\ell)} \mathbf{q}_{(r)}\right)=\emptyset$ for some $\ell, r \in \mathbb{N}^{\star}$.

(i) If $\mathrm{h}(\mathbf{u})=\mathrm{h}(\mathbf{v})$ and $\mathrm{t}(\mathbf{u}) \neq \mathrm{t}(\mathbf{v})$, then $\mathrm{C}_{0}^{1}\left\{(6),(7), \mathbf{u q}_{(r)} \approx \mathbf{v q}_{(r)}\right\}=\mathrm{C}_{0}^{1}\left\{(7), \rho_{r}\right\}$.

(ii) If $\mathrm{h}(\mathbf{u}) \neq \mathrm{h}(\mathbf{v})$ and $\mathrm{t}(\mathbf{u})=\mathrm{t}(\mathbf{v})$, then $\mathrm{C}_{0}^{1}\left\{(6),(7), \mathbf{p}_{(\ell)} \mathbf{u} \approx \mathbf{p}_{(\ell)} \mathbf{v}\right\}=\mathrm{C}_{0}^{1}\left\{(6), \lambda_{\ell}\right\}$.

(iii) If $\mathrm{h}(\mathbf{u}) \neq \mathrm{h}(\mathbf{v})$ and $\mathrm{t}(\mathbf{u}) \neq \mathrm{t}(\mathbf{v})$, then $\mathrm{C}_{0}^{1}\left\{(6),(7), \mathbf{p}_{(\ell)} \mathbf{u} \mathbf{q}_{(r)} \approx \mathbf{p}_{(\ell)} \mathbf{v} \mathbf{q}_{(r)}\right\}=\mathrm{C}_{0}^{1}\left\{\lambda_{\ell}, \rho_{r}\right\}$.

Proof. (i) Suppose that $\mathrm{h}(\mathrm{u})=\mathrm{h}(\mathrm{v})$ and $\mathrm{t}(\mathrm{u})=x \neq y=\mathrm{t}(\mathrm{v})$. Since con $(\mathbf{u})=\mathrm{con}(\mathrm{v})$ by Lemma 4.1(iii), it follows from Lemma $2.2(i)$ that the identity $\mathbf{u} \approx \mathbf{v}$ does not delete to any identity in $\{(2),(3)\}$. Hence

$$
\mathbf{u}=\operatorname{axb} y \mathbf{c} x \quad \text { and } \quad \mathbf{v}=\mathbf{d} y \mathbf{e x f} y
$$

for some $\mathbf{a}, \mathbf{b}, \mathbf{c}, \mathbf{d}, \mathbf{e}, \mathbf{f} \in \mathcal{X}^{*}$ with $y \in \operatorname{con}(\mathbf{a b}) \backslash \operatorname{con}(\mathbf{c})$ and $x \in \operatorname{con}(\mathbf{d e}) \backslash$ con $(\mathbf{f})$. The deduction $\mathbf{u q}_{(r)} \approx \mathbf{v q}_{(r)} \Vdash \rho_{r}$ can be obtained by following the proof of Lemma 5.5(i). Hence

$$
\mathbf{C}_{0}^{1}\left\{(6),(7), \mathbf{u q}_{(r)} \approx \mathbf{v q}_{(r)}\right\}=\mathbf{C}_{0}^{1}\left\{(6),(7), \mathbf{u q}_{(r)} \approx \mathbf{v q}_{(r)}, \rho_{r}\right\}
$$

The deduction $\left\{(6), \rho_{r}\right\} \Vdash \mathbf{u q}_{(r)} \approx \mathbf{u}^{\prime} \mathbf{q}_{(r)}$, where $\mathbf{u}^{\prime}=\mathbf{a} \mathbf{x} \mathbf{b c x y}$, can also be obtained by following the proof of Lemma 5.5(i). Hence

$$
\mathbf{C}_{0}^{1}\left\{(6),(7), \mathbf{u q}_{(r)} \approx \mathbf{v} \mathbf{q}_{(r)}, \rho_{r}\right\}=\mathbf{C}_{0}^{1}\left\{(6),(7), \mathbf{u}^{\prime} \mathbf{q}_{(r)} \approx \mathbf{v} \mathbf{q}_{(r)}, \rho_{r}\right\}
$$

Now since $\mathbf{u}^{\prime} \approx \mathbf{v}$ is a quadratic, connected identity of the variety $A_{0}^{1}$ such that $h\left(\mathbf{u}^{\prime}\right)=\mathrm{h}(\mathbf{v})$ and $\mathrm{t}\left(\mathbf{u}^{\prime}\right)=\mathrm{t}(\mathbf{v})$, the deduction $\{(6),(7)\} \Vdash \mathbf{u}^{\prime} \approx \mathbf{v}$ holds by Lemma 5.4(iii). Further, the deduction $\rho_{r} \Vdash(6)$ holds by Lemma 5.3(ii). Therefore

$$
\mathrm{C}_{0}^{1}\left\{(6),(7), \mathbf{u}^{\prime} \mathbf{q}_{(r)} \approx \mathbf{v} \mathbf{q}_{(r)}, \rho_{r}\right\}=\mathrm{C}_{0}^{1}\left\{(7), \rho_{r}\right\}
$$

The result is now obtained by combining (10a), (10b), and (10c).

(ii) This is symmetrical to part (i).

(iii) This can be established with slight modifications to arguments in the proofs of parts (i) and (ii). But complete details are given here for the sake of clarity, since some details of the proof of part (i) were omitted due to similarities with the proof of Lemma 5.5(i).

Suppose that $\mathrm{h}(\mathbf{u})=x \neq y=\mathrm{h}(\mathbf{v})$ and $\mathrm{t}(\mathbf{u})=z \neq t=\mathrm{t}(\mathbf{v})$. Since con $(\mathbf{u})=$ con $(\mathbf{v})$ by Lemma 4.1(iii), it follows from Lemma 2.2(i) that the identity $\mathbf{u} \approx \mathbf{v}$ does not delete to any identity in $\{(2),(3)\}$. The assumption $\mathrm{h}(\mathbf{u})=x \neq y=\mathrm{h}(\mathbf{v})$ implies that

$$
\mathbf{u}=x \mathbf{a}_{1} y \mathbf{b}_{1} x \mathbf{c}_{1} \quad \text { and } \quad \mathbf{v}=y \mathbf{a}_{2} x \mathbf{b}_{2} y \mathbf{c}_{2}
$$


for some $\mathbf{a}_{i}, \mathbf{b}_{i}, \mathbf{c}_{i} \in \mathcal{X}^{*}$ with $y \in \operatorname{con}\left(\mathbf{b}_{1} \mathbf{c}_{1}\right) \backslash \operatorname{con}\left(\mathbf{a}_{1}\right)$ and $x \in \operatorname{con}\left(\mathbf{b}_{2} \mathbf{c}_{2}\right) \backslash \operatorname{con}\left(\mathbf{a}_{2}\right)$. The assumption $\mathrm{t}(\mathbf{u})=z \neq t=\mathrm{t}(\mathbf{v})$ implies that

$$
\mathbf{u}=\mathbf{d}_{1} z \mathbf{e}_{1} t \mathbf{f}_{1} z \quad \text { and } \quad \mathbf{v}=\mathbf{d}_{2} t \mathbf{e}_{2} z \mathbf{f}_{2} t
$$

for some $\mathbf{d}_{i}, \mathbf{e}_{i}, \mathbf{f}_{i} \in \mathcal{X}^{*}$ with $t \in \operatorname{con}\left(\mathbf{d}_{1} \mathbf{e}_{1}\right) \backslash \operatorname{con}\left(\mathbf{f}_{1}\right)$ and $z \in \operatorname{con}\left(\mathbf{d}_{2} \mathbf{e}_{2}\right) \backslash \operatorname{con}\left(\mathbf{f}_{2}\right)$. Let $\varphi$ be the substitution $h \mapsto x$ for all $h \in \operatorname{con}\left(\mathbf{u q}_{(r)}\right) \backslash\{x, y\}$. Then $\left(\left(\mathbf{p}_{(\ell)} \mathbf{u q}_{(r)}\right) \varphi\right) y x \stackrel{(\star)}{\approx} \mathbf{p}_{(\ell)} x y^{2} x$ and $\left(\left(\mathbf{p}_{(\ell)} \mathbf{v q}_{(r)}\right) \varphi\right) y x \stackrel{(\star)}{\approx} \mathbf{p}_{(\ell)} y x y x$ imply the deduction $\mathbf{p}_{(\ell)} \mathbf{u q}_{(r)} \approx \mathbf{p}_{(\ell)} \mathbf{v} \mathbf{q}_{(r)} \Vdash \lambda_{\ell}$. Let $\chi$ be the substitution $h \mapsto z$ for all $h \in \operatorname{con}\left(\mathbf{p}_{(\ell)} \mathbf{u}\right) \backslash\{z, t\}$. Then $z t\left(\left(\mathbf{p}_{(\ell)} \mathbf{u} \mathbf{q}_{(r)}\right) \chi\right) \stackrel{(\star)}{\approx} z t^{2} z \mathbf{q}_{(r)}$ and $z t\left(\left(\mathbf{p}_{(\ell)} \mathbf{v} \mathbf{q}_{(r)}\right) \chi\right) \stackrel{(\star)}{\approx} z t z t \mathbf{q}_{(r)}$ imply the deduction $\mathbf{p}_{(\ell)} \mathbf{u} \mathbf{q}_{(r)} \approx \mathbf{p}_{(\ell)} \mathbf{v} \mathbf{q}_{(r)} \Vdash \rho_{r}$. Hence

$$
\mathbf{C}_{0}^{1}\left\{(6),(7), \mathbf{p}_{(\ell)} \mathbf{u} \mathbf{q}_{(r)} \approx \mathbf{p}_{(\ell)} \mathbf{v} \mathbf{q}_{(r)}\right\}=\mathrm{C}_{0}^{1}\left\{(6),(7), \mathbf{p}_{(\ell)} \mathbf{u} \mathbf{q}_{(r)} \approx \mathbf{p}_{(\ell)} \mathbf{v} \mathbf{q}_{(r)}, \lambda_{\ell}, \rho_{r}\right\}
$$

Consider the word $\mathbf{u}$ written in the form $\mathbf{u}=x \mathbf{a}_{1} y \mathbf{b}_{1} x \mathbf{c}_{1}$ above. Note that

(a) if some simple letter $h$ of the word $\mathbf{u}$ belongs to the factor $\mathbf{a}_{1}$, then the identity $\mathbf{u} \approx \mathbf{v}$ deletes to the identity $h y^{2} \approx \mathbf{w}$ for some $\mathbf{w} \in\left\{y h y, y^{2} h\right\}$

(b) if both occurrences of some non-simple letter $h$ of the word $\mathbf{u}$ belong to the factor $\mathbf{a}_{1}$, then the identity $\mathbf{u} \approx \mathbf{v}$ deletes to the identity $h^{2} y^{2} \approx \mathrm{w}$ for some $\mathrm{w} \in\left\{y h^{2} y, y h y h, y^{2} h^{2}\right\}$.

In both (a) and (b), the identity $\mathbf{u} \approx \mathbf{v}$ deletes to some identity from $\{(2),(3)\}$, contradicting an earlier observation. Thus neither (a) nor (b) is possible, whence each letter in the factor $\mathbf{a}_{1}$ is the first occurrence of some non-simple letter of the quadratic word $\mathbf{u}$, that is, each letter in $\mathbf{a}_{1}$ has a second occurrence somewhere in $\mathbf{b}_{1}$ or $\mathbf{c}_{1}$. Therefore

$$
\begin{aligned}
& \mathbf{p}_{(\ell)} \mathbf{u q}_{(r)} \stackrel{(\star)}{\approx} \mathbf{p}_{(\ell)}\left(x \mathbf{a}_{1} y^{2} \mathbf{a}_{1} x\right) \mathbf{b}_{1} x \mathbf{c}_{1} \mathbf{q}_{(r)} \stackrel{(7)}{\approx} \mathbf{p}_{(\ell)} x y \mathbf{a}_{1} y \mathbf{a}_{1} x \mathbf{b}_{1} x \mathbf{c}_{1} \mathbf{q}_{(r)} \stackrel{(\star)}{\approx}\left(\mathbf{p}_{(\ell)} x y^{2} x\right) \mathbf{a}_{1} \mathbf{b}_{1} x \mathbf{c}_{1} \mathbf{q}_{(r)} \\
& \stackrel{\lambda_{\ell}}{\approx} \mathbf{p}_{(\ell)} y x y x \mathbf{a}_{1} \mathbf{b}_{1} x \mathbf{c}_{1} \mathbf{q}_{(r)} \stackrel{(\star)}{\approx} \mathbf{p}_{(\ell)} y x \mathbf{a}_{1} \mathbf{b}_{1} x \mathbf{c}_{1} \mathbf{q}_{(r)}=\mathbf{p}_{(\ell)} \mathbf{u}^{\prime} \mathbf{q}_{(r)},
\end{aligned}
$$

that is, the deduction

$$
\left\{(7), \lambda_{\ell}\right\} \Vdash \mathbf{p}_{(\ell)} \mathbf{u q}_{(r)} \approx \mathbf{p}_{(\ell)} \mathbf{u}^{\prime} \mathbf{q}_{(r)}
$$

holds where $\mathbf{u}^{\prime}=y \times \mathbf{a}_{1} \mathbf{b}_{1} \times \mathbf{c}_{1}$ is a connected word such that $\mathrm{h}\left(\mathbf{u}^{\prime}\right)=\mathrm{h}(\mathbf{v})$ and $\mathrm{t}\left(\mathbf{u}^{\prime}\right)=\mathrm{t}(\mathbf{u})$. Now consider the word $\mathbf{v}$ written in the form $\mathbf{v}=\mathbf{d}_{2} t \mathbf{e}_{2} z \mathbf{f}_{2} t$ above. By a symmetrical argument, the deduction

$$
\left\{(6), \lambda_{\ell}\right\} \Vdash \mathbf{p}_{(\ell)} \mathbf{v} \mathbf{q}_{(r)} \approx \mathbf{p}_{(\ell)} \mathbf{v}^{\prime} \mathbf{q}_{(r)}
$$

is obtained where $\mathbf{v}^{\prime}=\mathbf{d}_{2} t \mathbf{e}_{2} \mathbf{f}_{2} t z$ is a connected word such that $h\left(\mathbf{v}^{\prime}\right)=h(\mathbf{v})$ and $t\left(\mathbf{v}^{\prime}\right)=t(\mathbf{u})$. It follows from (11a), (11b) and (11c) that

$$
\mathrm{C}_{0}^{1}\left\{(6),(7), \mathbf{p}_{(\ell)} \mathbf{u} \mathbf{q}_{(r)} \approx \mathbf{p}_{(\ell)} \mathbf{v} \mathbf{q}_{(r)}\right\}=\mathrm{C}_{0}^{1}\left\{(6),(7), \mathbf{p}_{(\ell)} \mathbf{u}^{\prime} \mathbf{q}_{(r)} \approx \mathbf{p}_{(\ell)} \mathbf{v}^{\prime} \mathbf{q}_{(r)}, \lambda_{\ell}, \rho_{r}\right\}
$$

Since $\mathbf{u}^{\prime} \approx \mathbf{v}^{\prime}$ is a quadratic, connected identity of $\mathrm{A}_{0}^{1}$ with $\mathrm{h}\left(\mathbf{u}^{\prime}\right)=\mathrm{h}\left(\mathbf{v}^{\prime}\right)$ and $\mathrm{t}\left(\mathbf{u}^{\prime}\right)=\mathrm{t}\left(\mathbf{v}^{\prime}\right)$, the deduction $\{(6),(7)\} \Vdash \mathbf{u}^{\prime} \approx \mathbf{v}^{\prime}$ holds by Lemma 5.4(iii); this deduction, together with the deduction $\left\{\lambda_{\ell}, \rho_{r}\right\} \Vdash\{(6),(7)\}$ in Lemma 5.3, imply that

$$
\mathbf{C}_{0}^{1}\left\{(6),(7), \mathbf{p}_{(\ell)} \mathbf{u}^{\prime} \mathbf{q}_{(r)} \approx \mathbf{p}_{(\ell)} \mathbf{v}^{\prime} \mathbf{q}_{(r)}, \lambda_{\ell}, \rho_{r}\right\}=\mathbf{C}_{0}^{1}\left\{\lambda_{\ell}, \rho_{r}\right\}
$$

The result is now obtained by combining (11d) and (11e).

\section{Lemma 5.7.}

Let $\sigma: \mathbf{p}_{(\ell)} \mathbf{u q}_{(r)} \approx \mathbf{p}_{(\ell)} \mathbf{v q}_{(r)}$ be the identity (4) that satisfies conditions (1†)-(6†) in Lemma 4.2. Suppose that the variety $\mathrm{A}_{0}^{1}$ satisfies the identity $\sigma$. Then $\mathrm{C}_{0}^{1}\{\sigma\}=\mathrm{C}_{0}^{1}\left(\Sigma_{1} \cup \Sigma_{2}\right)$ for some $\Sigma_{1} \subseteq\{(6),(7)\}$ and some $\Sigma_{2} \subseteq\left\{\lambda_{\ell}, \rho_{r}\right\}$. 
Proof. Since $A_{0}^{1}$ is a monoid, it follows from condition (3†) of Lemma 4.2 that the variety $\mathrm{A}_{0}^{1}$ also satisfies the identity $\mathbf{u} \approx \mathbf{v}$. There are four cases determined by the conditions ini $(\mathbf{u})=\operatorname{ini}(\mathbf{v})$ and $\operatorname{fin}(\mathbf{u})=\operatorname{fin}(\mathbf{v})$.

Case 1: ini $(\mathbf{u})=\operatorname{ini}(\mathbf{v})$ and fin $(\mathbf{u})=$ fin $(\mathbf{v})$. By Lemma 2.1, the identity $\sigma$ is satisfied by the monoids $L_{2}^{1}$ and $R_{2}^{1}$. Since $C_{0}^{1}=A_{0}^{1} \vee L_{2}^{1} \vee R_{2}^{1}$ by Lemma 3.2, the identity $\sigma$ is also satisfied by the variety $C_{0}^{1}$. Hence $C_{0}^{1}\{\sigma\}=C_{0}^{1}$.

Case 2: $\operatorname{ini}(\mathbf{u})=\operatorname{ini}(\mathbf{v})$ and fin $(\mathbf{u}) \neq$ fin $(\mathbf{v})$. Since $h(\mathbf{u})=\mathrm{h}(\mathbf{v})$, it follows from condition (5†) of Lemma 4.2 that the identity $\sigma$ is $\mathbf{u q}_{(r)} \approx \mathbf{v q}_{(r)}$. By Lemma 2.1(ii), the identity $\sigma$ is not satisfied by the monoid $R_{2}^{1}$. Hence

(a) $\mathbf{C}_{0}^{1}\{\sigma\}=\mathbf{C}_{0}^{1}\left\{(6), \mathbf{u q}_{(r)} \approx \mathbf{v} \mathbf{q}_{(r)}\right\}$

by Lemma 5.2(i). If $\mathrm{t}(\mathbf{u}) \neq \mathrm{t}(\mathbf{v})$, then $\mathrm{C}_{0}^{1}\left\{\sigma \stackrel{(\mathrm{a})}{=} \mathrm{C}_{0}^{1}\left\{(6), \mathbf{u} \mathbf{q}_{(r)} \approx \mathbf{v} \mathbf{q}_{(r)}\right\}=\mathrm{C}_{0}^{1}\left\{\rho_{r}\right\}\right.$ by Lemma 5.5(i). If $\mathrm{t}(\mathbf{u})=\mathrm{t}(\mathbf{v})$, then the identity $\sigma$ is $\mathbf{u} \approx \mathbf{v}$ by condition $(6+)$ of Lemma 4.2, whence $\mathrm{C}_{0}^{1}\{\sigma\} \stackrel{(\mathrm{a})}{=} \mathrm{C}_{0}^{1}\{(6), \mathbf{u} \approx \mathbf{v}\}=\mathrm{C}_{0}^{1}(6)$ by Lemma 5.4(i).

Case 3: ini $(\mathbf{u}) \neq \operatorname{ini}(\mathbf{v})$ and fin $(\mathbf{u})=$ fin $(\mathbf{v})$. By an argument that is symmetrical to Case 2, the variety $\mathrm{C}_{0}^{1}\{\sigma\}$ is either $\mathrm{C}_{0}^{1}\left\{\lambda_{\ell}\right\}$ or $\mathrm{C}_{0}^{1}(7)$.

Case 4: ini $(\mathbf{u}) \neq$ ini $(\mathbf{v})$ and $\operatorname{fin}(\mathbf{u}) \neq$ fin $(\mathbf{v})$. Then

(b) $\mathbf{C}_{0}^{1}\{\sigma\}=\mathbf{C}_{0}^{1}\left\{(6),(7), \mathbf{p}_{(\ell)} \mathbf{u q} \mathbf{q}_{(r)} \approx \mathbf{p}_{(\ell)} \mathbf{v} \mathbf{q}_{(r)}\right\}$

by Lemmas 2.1 and 5.2. There are four subcases determined by the conditions $h(\mathbf{u})=\mathrm{h}(\mathbf{v})$ and $\mathrm{t}(\mathbf{u})=\mathrm{t}(\mathbf{v})$.

4.1: $\mathrm{h}(\mathbf{u})=\mathrm{h}(\mathbf{v})$ and $\mathrm{t}(\mathbf{u})=\mathrm{t}(\mathbf{v})$. Then the identity $\sigma$ is $\mathbf{u} \approx \mathbf{v}$ by conditions (5†) and (6†) in Lemma 4.2. Hence $\mathrm{C}_{0}^{1}\{\sigma\} \stackrel{(b)}{=} \mathrm{C}_{0}^{1}\{(6),(7), \mathbf{u} \approx \mathrm{v}\}=\mathrm{C}_{0}^{1}\{(6),(7)\}$ by Lemma 5.4(iii).

4.2: $\mathrm{h}(\mathbf{u})=\mathrm{h}(\mathbf{v})$ and $\mathrm{t}(\mathbf{u}) \neq \mathrm{t}(\mathbf{v})$. Then the identity $\sigma$ is $\mathbf{u q}_{(r)} \approx \mathbf{v} \mathbf{q}_{(r)}$ by conditions (5†) in Lemma 4.2. Hence $\mathrm{C}_{0}^{1}\{\sigma\} \stackrel{(\mathrm{b})}{=} \mathrm{C}_{0}^{1}\left\{(6),(7), \mathbf{u q}_{(r)} \approx \mathbf{v q}_{(r)}\right\}=\mathrm{C}_{0}^{1}\left\{(7), \rho_{r}\right\}$ by Lemma 5.6(i).

4.3: $\mathrm{h}(\mathbf{u}) \neq \mathrm{h}(\mathbf{v})$ and $\mathrm{t}(\mathbf{u})=\mathrm{t}(\mathbf{v})$. This is symmetrical to Subcase 4.2 so that $\mathrm{C}_{0}^{1}\{\sigma\}=\mathrm{C}_{0}^{1}\left\{(6), \lambda_{\ell}\right\}$.

4.4: $\mathrm{h}(\mathbf{u}) \neq \mathrm{h}(\mathbf{v})$ and $\mathrm{t}(\mathbf{u}) \neq \mathrm{t}(\mathbf{v})$. Then $\mathrm{C}_{0}^{1}\{\sigma\} \stackrel{(\mathrm{b})}{=} \mathrm{C}_{0}^{1}\left\{(6),(7), \mathbf{p}_{(\ell)} \mathbf{u} \mathbf{q}_{(r)} \approx \mathbf{p}_{(\ell)} \mathbf{v} \mathbf{q}_{(r)}\right\}=\mathrm{C}_{0}^{1}\left\{\lambda_{\ell}, \rho_{r}\right\}$ by Lemma 5.6(iii).

\section{Proposition 5.8.}

Any variety in the interval $\left[\mathrm{A}_{0}^{1}, \mathrm{C}_{0}^{1}\right]$ is finitely based.

Proof. Let $\mathrm{V}$ be any variety in the interval $\left[\mathrm{A}_{0}^{1}, \mathrm{C}_{0}^{1}\right]$. Then $\mathrm{V}=\mathrm{C}_{0}^{1} \Sigma$ for some set $\Sigma$ of identities of the variety $\mathrm{A}_{0}^{1}$. By Lemma 4.2, the identities in the set $\Sigma$ can be chosen to be of the form (4) that satisfy conditions (1†)-(6†).

Let $\sigma: \mathbf{p}_{(\ell)} \mathbf{u} \mathbf{q}_{(r)} \approx \mathbf{p}_{(\ell)} \mathbf{v} \mathbf{q}_{(r)}$ be any identity from the set $\Sigma$. By condition (3†) of Lemma 4.2, the variety $\mathrm{A}_{0}^{1}$ satisfies the identity $\mathbf{u} \approx \mathbf{v}$. Then it follows from Lemma 5.7 that $\mathbf{C}_{0}^{1}\{\sigma\}=\mathrm{C}_{0}^{1}\left(\Sigma_{1}^{\sigma} \cup \Sigma_{2}^{\sigma}\right)$ for some $\Sigma_{1}^{\sigma} \subseteq\{(6)$, (7) $\}$ and some $\Sigma_{2}^{\sigma} \subseteq\left\{\lambda_{\ell}, \rho_{r}\right\}$. Since the identity $\sigma$ is arbitrarily chosen from the set $\Sigma$, repeating the same argument on every identity in $\Sigma$ yields $\mathrm{C}_{0}^{1} \Sigma=\mathrm{C}_{0}^{1}\left(\Sigma_{1} \cup \Sigma_{2}\right)$ for some $\Sigma_{1} \subseteq\{(6),(7)\}$ and some $\Sigma_{2} \subseteq\left\{\lambda_{n}, \rho_{n} \mid n \in \mathbb{N}^{\star}\right\}$. It follows from Lemma 5.3 that $\mathrm{V}=\mathrm{C}_{0}^{1}\left(\Sigma_{1} \cup \Sigma_{2}^{\prime}\right)$ for some finite subset $\Sigma_{2}^{\prime}$ of $\Sigma_{2}$.

\subsection{Varieties in $\left[A_{0} \vee B_{0}^{1}, C_{0}^{1}\right]$ not containing $A_{0}^{1}$}

The main result of this subsection, Proposition 5.12, establishes the finite basis property of every variety in the interval $\left[A_{0} \vee B_{0}^{1}, C_{0}^{1}\right]$ that does not contain the variety $A_{0}^{1}$.

\section{Lemma 5.9.}

Let $\mathrm{V}$ be any subvariety of $\mathrm{C}_{0}^{1}$ such that $A_{0}^{1} \notin \mathrm{V}$. Then the variety $\mathrm{V}$ satisfies the identity

$$
h x y x y h \approx h x^{2} y^{2} h
$$


Proof. It follows from Torlopova [21] that the identity $x y x y \approx x^{2} y^{2}$ defines the subvariety of $\mathrm{C}_{0}^{1}$ that is largest with respect to not containing the semigroup $A_{0}$. Suppose that $A_{0}^{1} \notin \mathrm{V}$. Let $\varphi$ be the substitution $z \mapsto h^{2} z h^{2}$ for all $z \in \mathcal{X}$. Then it follows from Lee [8, Theorem 2] that the variety $V$ satisfies the identity $(x y x y) \varphi \approx\left(x^{2} y^{2}\right) \varphi$. It is easy to deduce that the variety $\mathrm{V}$ also satisfies the identity (12).

Let $x$ and $y$ be any distinct non-simple letters of a quadratic word $\mathbf{w}$. If $\mathbf{w} \in \mathcal{X}^{*} x \mathcal{X}^{*} x y \mathcal{X}^{*} y \mathcal{X}^{*}$, then $x$ and $y$ form a standoff pair in w. A quadratic word with no standoff pairs is said to be peaceful.

Lemma 5.10.

Let $\mathbf{w}$ be any connected, quadratic word. Then the deduction (12) $\Vdash \mathbf{w} \approx \mathbf{w}^{\pi}$ holds for some peaceful, connected, quadratic word $\mathbf{w}^{\pi}$.

Proof. Suppose that the letters $x$ and $y$ form a standoff pair in $\mathbf{w}$. Then $\mathbf{w}=\mathbf{a} x \mathbf{b} x y \mathbf{c} y \mathbf{d}$ for some $\mathbf{a}, \mathbf{b}, \mathbf{c}, \mathbf{d} \in \mathcal{X}^{*}$. Since the word $\mathbf{w}$ is connected, its factors $\mathbf{a} x \mathbf{b} x$ and $y \mathbf{c} y \mathbf{d}$ share some common letter $h$. Further, the word $\mathbf{w}$ is quadratic so that the letter $h$ is neither $x$ nor $y$. Therefore

$$
\mathbf{w} \stackrel{(\star)}{\approx} \mathbf{a} x \mathbf{b} h x^{2} y^{2} h \mathrm{c} y \mathbf{d} \stackrel{(12)}{\approx} \mathrm{a} x \mathbf{b} h x y x y h c y \mathbf{d} \stackrel{(\star)}{\approx} \mathrm{a} x \mathrm{~b} y x \mathrm{c} y \mathbf{d}=\mathbf{w}_{x, y}
$$

that is, the identities $\{(\star),(12)\}$ can be used to convert the word $\mathbf{w}$ into a word $\mathbf{w}_{x, y}$ in which the letters $x$ and $y$ no longer form a standoff pair. In other words, the identities $\{(\star),(12)\}$ reconciled the standoff pair $x$ and $y$. Observe that in the process of reconciliating a standoff pair $x$ and $y$,

- the distance between the two occurrences of $x$ is increased by one;

- the distance between the two occurrences of $y$ is increased by one;

- the distance between the two occurrences of any other non-simple letter remains unchanged.

Therefore the separation degree of the resulting word $\mathbf{w}_{x, y}$ is at least two greater than the separation degree of $\mathbf{w}$. It is easy to see that the word $\mathbf{w}_{x, y}$ is connected and quadratic such that $\mathbf{w} \stackrel{\circ}{=} \mathbf{w}_{x, y}$.

There are at most $|\mathbf{w}|$ ! words that are $\stackrel{\circ}{=}$-related to the word $\mathbf{w}$. Among all these $|\mathbf{w}|$ ! words, one must possess the greatest possible separation degree, say $d$. By the above observations, the process of reconciliation can only be repeated on $\mathbf{w}$ at most $\lfloor d / 2\rfloor$ times. When no more reconciliation can be performed, the word is then the required peaceful, connected, quadratic word $\mathbf{w}^{\pi}$.

Lemma 5.11.

Let $\mathbf{u}$ and $\mathbf{v}$ be peaceful, connected, quadratic words such that the identity $\mathbf{u} \approx \mathbf{v}$ does not delete to any identity in (2). Then the identity $\mathbf{u} \approx \mathbf{v}$ does not delete to any identity in (3).

Proof. Working toward a contradiction, suppose that the identity $\mathbf{u} \approx \mathbf{v}$ deletes to some identity in (3). By symmetry, it suffices to assume that the identity $\mathbf{u} \approx \mathbf{v}$ deletes to one of the following identities: $x y^{2} x \approx x^{2} y^{2}, x y x y \approx x^{2} y^{2}$, $y x y x \approx x^{2} y^{2}$, and $y^{2} x^{2} \approx x^{2} y^{2}$. Hence the word $\mathrm{u}$ is either

$$
\text { axbycydxe or axbycxdye or aybxcydxe or aybycxdxe }
$$

for some a, b, c, d, e $\in \mathcal{X}^{*}$ with $x, y \notin$ con (abcde), and

$$
v=\mathbf{a}^{\prime} x \mathbf{b}^{\prime} x \mathbf{c}^{\prime} y \mathbf{d}^{\prime} y \mathbf{e}^{\prime}
$$

for some $\mathbf{a}^{\prime}, \mathbf{b}^{\prime}, \mathbf{c}^{\prime}, \mathbf{d}^{\prime}, \mathbf{e}^{\prime} \in \mathcal{X}^{*}$ with $x, y \notin \operatorname{con}\left(\mathbf{a}^{\prime} \mathbf{b}^{\prime} \mathbf{c}^{\prime} \mathbf{d}^{\prime} \mathbf{e}^{\prime}\right)$. If $\mathbf{c}^{\prime}=\emptyset$, then the letters $x$ and $y$ form a standoff pair in the word $\mathbf{v}$. If $\mathbf{c}^{\prime} \neq \varnothing$ and every letter in the factor $\mathbf{c}^{\prime}$ is non-simple in the word $\mathbf{v}$, then $\mathbf{v}$ contains some standoff pair. If $\mathbf{c}^{\prime} \neq \varnothing$ and some letter in the factor $\mathbf{c}^{\prime}$ is simple in the word $\mathbf{v}$, then it is easy to show that the identity $\mathbf{u} \approx \mathbf{v}$ deletes to some identity in (2). 
Proposition 5.12.

Any variety in the interval $\left[\mathrm{A}_{0} \vee \mathrm{B}_{0}^{1}, \mathrm{C}_{0}^{1}\right]$ that does not contain the variety $\mathrm{A}_{0}^{1}$ is finitely based.

Proof. Let $\vee$ be any variety in the interval $\left[\mathrm{A}_{0} \vee \mathrm{B}_{0}^{1}, \mathrm{C}_{0}^{1}\right]$ such that $\mathrm{A}_{0}^{1} \nsubseteq \mathrm{V}$. Then it follows from Lemma 5.9 that $\mathrm{V}=\mathrm{C}_{0}^{1}((12) \cup \Sigma)$ for some set $\Sigma$ of identities of the variety $\mathrm{A}_{0} \vee \mathrm{B}_{0}^{1}$. By Lemma 4.2, the identities in the set $\Sigma$ can be chosen to be of the form (4) that satisfy conditions (1†)-(6†).

Let $\sigma: \mathbf{p}_{(\ell)} \mathbf{u} \mathbf{q}_{(r)} \approx \mathbf{p}_{(\ell)} \mathbf{v} \mathbf{q}_{(r)}$ be any identity from the set $\Sigma$. By condition (3†) of Lemma 4.2,

(a) $\mathrm{B}_{0}^{1} \vDash \mathbf{u} \approx \mathrm{v}$.

Since the words $\mathbf{u}$ and $\mathbf{v}$ are connected and quadratic by condition (2†) of Lemma 4.2, it follows from Lemma 5.10 that the deduction

(b) (12) $\Vdash\left\{\mathbf{u} \approx \mathbf{u}^{\pi}, \mathbf{v} \approx \mathbf{v}^{\pi}\right\}$

holds for some peaceful, connected, quadratic words $\mathbf{u}^{\pi}$ and $\mathbf{v}^{\pi}$. Hence

(c) $\mathrm{C}_{0}^{1}\{(12), \sigma\}=\mathrm{C}_{0}^{1}\left\{(12), \sigma^{\pi}\right\}$

where $\sigma^{\pi}$ is the identity $\mathbf{p}_{(\ell)} \mathbf{u}^{\pi} \mathbf{q}_{(r)} \approx \mathbf{p}_{(\ell)} \mathbf{v}^{\pi} \mathbf{q}_{(r)}$. By Lemma 2.2(ii), the variety $\mathrm{B}_{0}^{1}$ satisfies the identity (12). Therefore it follows from (a) and (b) that the variety $\mathbf{B}_{0}^{1}$ satisfies the identity $\mathbf{u}^{\pi} \approx \mathbf{v}^{\pi}$, whence by Lemma 2.2(ii), the identity $\mathbf{u}^{\pi} \approx \mathbf{v}^{\pi}$ does not delete to any identity in (2). By Lemma 5.11, the identity $\mathbf{u}^{\pi} \approx \mathbf{v}^{\pi}$ also does not delete to any identity in (3). Hence by Lemma 2.2(i), the variety $A_{0}^{1}$ satisfies the identity $\mathbf{u}^{\pi} \approx \mathbf{v}^{\pi}$ so that

(d) $\mathrm{A}_{0}^{1} \vDash \sigma^{\pi}$.

Since the identity $\sigma$ is arbitrarily chosen from the set $\Sigma$, the construction of $\sigma^{\pi}$ from $\sigma$ can be repeated on every other identity in $\Sigma$ to obtain the set $\Sigma^{\pi}=\left\{\sigma^{\pi} \mid \sigma \in \Sigma\right\}$ where each identity in $\Sigma^{\pi}$ satisfies (c) and (d). Now the variety $\mathrm{C}_{0}^{1} \Sigma^{\pi}$ belongs to the interval $\left[\mathrm{A}_{0}^{1}, \mathrm{C}_{0}^{1}\right]$ and so is finitely based by Proposition 5.8. Therefore the variety $\mathrm{V}=\mathrm{C}_{0}^{1}((12) \cup \Sigma) \stackrel{(c)}{=} \mathrm{C}_{0}^{1}(12) \cap \mathrm{C}_{0}^{1} \Sigma^{\pi}$ is also finitely based.

\section{Proof of Theorem 3.3: subvarieties not containing $A_{0} \vee B_{0}^{1}$}

\section{Proposition 6.1.}

Any subvariety of $\mathrm{C}_{0}^{1}$ that does not contain the variety $\mathrm{A}_{0} \vee \mathrm{B}_{0}^{1}$ is finitely based.

Proof. Let $\mathrm{V}$ be any subvariety of $\mathrm{C}_{0}^{1}$ that does not contain the variety $\mathrm{A}_{0} \vee \mathrm{B}_{0}^{1}$. Then there are two cases depending on whether $\mathrm{A}_{0} \nsubseteq \mathrm{V}$ or $\mathrm{B}_{0}^{1} \nsubseteq \mathrm{V}$.

Case 1: $\mathrm{A}_{0} \not \subseteq \mathrm{V}$. Then the variety $\mathrm{V}$ satisfies the identity $x y x y \approx x^{2} y^{2}$, since this identity defines the subvariety of $\mathrm{C}_{0}^{1}$ that is largest with respect to not containing the semigroup $A_{0}$ [21]. Any variety that satisfies the identities $(\star)$ and $x y x y \approx x^{2} y^{2}$ is finitely based [28, Chapter 3]; see also Luo and Zhang [17].

Case 2: $\mathrm{A}_{0} \subseteq \mathrm{V}$ and $\mathrm{B}_{0}^{1} \nsubseteq \mathrm{V}$. There are two subcases depending on whether or not the variety $\mathrm{V}$ contains the subvariety $\mathrm{N}_{2}^{1}$ of $\mathrm{B}_{0}^{1}$.

2.1: $N_{2}^{1} \subseteq \mathrm{V}$. Then $\mathrm{V} \in\left[\mathrm{A}_{0} \vee \mathrm{N}_{2}^{1}, \mathrm{C}_{0}^{1}\right]$ and $\mathrm{B}_{0}^{1} \nsubseteq \mathrm{V}$, and $\mathrm{V}$ is shown to be finitely based in Subsection 6.1.

2.2: $\mathrm{N}_{2}^{1} \nsubseteq \mathrm{V}$. Then $\mathrm{V} \in\left[\mathrm{A}_{0}, \mathrm{C}_{0}^{1}\right]$ and $\mathrm{N}_{2}^{1} \nsubseteq \mathrm{V}$, and $\mathrm{V}$ is shown to be finitely based in Subsection 6.2.

\section{Lemma 6.2.}

Suppose that the identity $\mathbf{u} \approx \mathbf{v}$ deletes to the identity $\mathbf{u}^{\prime} \approx \mathbf{v}^{\prime}$. Then the deduction $\mathbf{u} \approx \mathbf{v} \Vdash h \mathbf{u}^{\prime} h \approx h \mathbf{v}^{\prime} h$ holds. 
Proof. Suppose that the identity $\mathbf{u}^{\prime} \approx \mathbf{v}^{\prime}$ is obtained from the identity $\mathbf{u} \approx \mathbf{v}$ by removing all occurrences of the letters $x_{1}, \ldots, x_{r}$. Denote by $\varphi$ the substitution $x_{i} \mapsto h$ for all $i \in\{1, \ldots, r\}$. Then

$$
\mathbf{u} \approx \mathbf{v} \Vdash h \mathbf{u}^{\prime} h \stackrel{(\star)}{\approx} h(\mathbf{u} \varphi) h \approx h(\mathbf{v} \varphi) h \stackrel{(\star)}{\approx} h \mathbf{v}^{\prime} h \vdash h \mathbf{u}^{\prime} h \approx h \mathbf{v}^{\prime} h
$$

\subsection{Varieties in $\left[A_{0} \vee N_{2}^{1}, C_{0}^{1}\right]$ not containing $B_{0}^{1}$.}

The main result of this subsection, Proposition 6.8, establishes the finite basis property of every variety in the interval $\left[A_{0} \vee N_{2}^{1}, C_{0}^{1}\right]$ that does not contain the variety $B_{0}^{1}$.

\section{Lemma 6.3.}

Let $\mathrm{V}$ be any variety in the interval $\left[\mathrm{A}_{0} \vee \mathrm{N}_{2}^{1}, \mathrm{C}_{0}^{1}\right]$ such that $B_{0}^{1} \notin \mathrm{V}$. Then the variety $\mathrm{V}$ satisfies one of the following identities:

$$
\begin{aligned}
h x y x h & \approx h x^{2} y h, \\
h x y x h & \approx h y x^{2} h, \\
h x^{2} y h & \approx h y x^{2} h, \\
h x y h & \approx h y x h .
\end{aligned}
$$

Proof. By assumption, some identity $\sigma$ of the variety $\mathrm{V}$ is not satisfied by the monoid $B_{0}^{1}$; by Remark 2.4, this identity can be chosen to be quadratic. By Lemmas 2.1(iii) and 2.2, the identity $\sigma$ deletes to one of the identities from (2) except $x^{2} \approx x$. The result now follows from Lemma 6.2 .

The following small semigroups are required in the proof of the next lemma:

$$
\begin{aligned}
I & =\left\langle a, b \mid a^{2}=b a=0, a b=a, b^{2}=b\right\rangle, \\
J & =\left\langle a, b \mid a^{2}=a b=0, b a=a, b^{2}=b\right\rangle, \\
K & =\left\langle a, b \mid a^{2}=b^{2}=b a=0\right\rangle .
\end{aligned}
$$

More information on the monoids $I^{1}, J^{1}$, and $K^{1}$ can be found in Lee [10, Section 1.3].

\section{Lemma 6.4.}

The varieties $C_{0}^{1}(13), C_{0}^{1}(14), C_{0}^{1}(15)$, and $C_{0}^{1}(16)$ constitute the following subsemilattice of the lattice of all semigroup varieties:

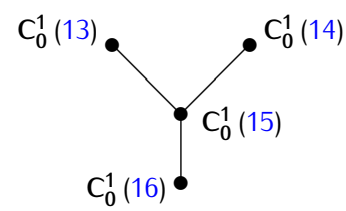

Proof. The inclusion $\mathrm{C}_{0}^{1}(15) \subseteq \mathrm{C}_{0}^{1}(13)$ holds since

$$
(15) \Vdash h x y x h \stackrel{(\star)}{\approx} h x\left(h y x^{2} h\right) \stackrel{(15)}{\approx} h x h x^{2} y h \stackrel{(\star)}{\approx} h x^{2} y h \vdash(13)
$$

By symmetry, the inclusion $C_{0}^{1}(15) \subseteq C_{0}^{1}(14)$ also holds. It is easy to verify that the inclusion $C_{0}^{1}(16) \subseteq C_{0}^{1}(15)$ and the intersection $C_{0}^{1}(13) \cap C_{0}^{1}(14)=C_{0}^{1}(15)$ hold. The varieties $C_{0}^{1}(13), C_{0}^{1}(14), C_{0}^{1}(15)$, and $C_{0}^{1}(16)$ are distinct because $I^{1} \in C_{0}^{1}(13) \backslash C_{0}^{1}(15), J^{1} \in C_{0}^{1}(14) \backslash C_{0}^{1}(15)$, and $K^{1} \in C_{0}^{1}(15) \backslash C_{0}^{1}(16)$. 


\section{Corollary 6.5 .}

Let $\mathrm{V}$ be any variety in the interval $\left[\mathrm{A}_{0} \vee \mathrm{N}_{2}^{1}, \mathrm{C}_{0}^{1}\right]$ such that $B_{0}^{1} \notin \mathrm{V}$. Then precisely one of the following conditions holds:

- $\mathrm{V} \subseteq \mathrm{C}_{0}^{1}(13)$ and $\mathrm{V} \nsubseteq \mathrm{C}_{0}^{1}(14)$;

- $\mathrm{V} \nsubseteq \mathrm{C}_{0}^{1}(13)$ and $\mathrm{V} \subseteq \mathrm{C}_{0}^{1}(14)$;

- $\mathrm{V} \subseteq \mathrm{C}_{0}^{1}(15)$ and $\mathrm{V} \nsubseteq \mathrm{C}_{0}^{1}(16)$;

- $\mathrm{V} \subseteq \mathrm{C}_{0}^{1}(16)$.

Proof. This follows from Lemmas 6.3 and 6.4 .

Let $x$ be any non-simple letter in a quadratic word $\mathbf{w}$, say

$$
\mathbf{w}=\mathbf{w}_{1} \times \mathbf{w}_{2} \times \mathbf{w}_{3}
$$

for some $\mathbf{w}_{1}, \mathbf{w}_{2}, \mathbf{w}_{3} \in \mathcal{X}^{*}$ such that $x \notin \operatorname{con}\left(\mathbf{w}_{1} \mathbf{w}_{2} \mathbf{w}_{3}\right)$. Then the letter $x$ is said to be left-disciplined in the word $\mathbf{w}$ if either $\mathbf{w}_{1}=\emptyset$ or the letter $t\left(\mathbf{w}_{1}\right)$ is the first occurrence of some non-simple letter in $\mathbf{w}$. A non-simple, quadratic word is said to be left-disciplined if all its non-simple letters are left-disciplined in it. Equivalently, a non-simple, quadratic word $\mathbf{w}$ is left-disciplined if and only if the first occurrences of non-simple letters in $\mathbf{w}$ form a prefix of $\mathbf{w}$.

Similarly, the letter $x$ in (17) is said to be right-disciplined in the word $w$ if either $w_{3}=\emptyset$ or the letter $h\left(w_{3}\right)$ is the second occurrence of some non-simple letter in $\mathbf{w}$. A non-simple, quadratic word is said to be right-disciplined if all its non-simple letters are right-disciplined in it. Equivalently, a non-simple, quadratic word $\mathbf{w}$ is right-disciplined if and only if the second occurrences of non-simple letters in $\mathbf{w}$ form a suffix of $\mathbf{w}$.

A word that is both left-disciplined and right-disciplined is said to be disciplined. Equivalently, a disciplined word $\mathrm{w}$ is non-simple, quadratic, and of the form

$$
\mathbf{w}=\mathrm{hw}^{\prime} \mathbf{t},
$$

where the prefix $\mathbf{h}$ consists of all first occurrences of non-simple letters in $\mathbf{w}$, the suffix $\mathbf{t}$ consists of all second occurrences of non-simple letters in $\mathbf{w}$, and the factor $\mathbf{w}^{\prime}$ consists of all simple letters of $\mathbf{w}$. Note that a disciplined word is necessarily connected and peaceful. The disciplined word $\mathbf{w}$ in (18) is said to be well-disciplined if the letters in the factor $\mathbf{w}^{\prime}$ are in alphabetical order.

\section{Lemma 6.6.}

Let $\mathbf{w}$ be any quadratic, connected word.

(i) The deduction (13) $\Vdash \mathbf{w} \approx \mathbf{w}^{\delta}$ holds for some right-disciplined, quadratic, connected word $\mathbf{w}^{\delta}$.

(ii) The deduction (14) $\Vdash \mathbf{w} \approx \mathbf{w}^{\delta}$ holds for some left-disciplined, quadratic, connected word $\mathbf{w}^{\delta}$.

(iii) The deduction (15) $\Vdash \mathbf{w} \approx \mathbf{w}^{\delta}$ holds for some disciplined, quadratic, connected word $\mathbf{w}^{\delta}$.

(iv) The deduction $(16) \Vdash \mathbf{w} \approx \mathbf{w}^{\delta}$ holds for some well-disciplined, quadratic, connected word $\mathbf{w}^{\delta}$.

Further, the relation $\mathbf{w} \stackrel{\circ}{=} \mathbf{w}^{\delta}$ holds in each of (i)-(iv).

Proof. (i) Let $w$ be any quadratic word and let $x$ be any non-simple letter in $w$ that is not right-disciplined. Then $\mathbf{w}=\mathbf{w}_{1} x \mathbf{w}_{2} x \mathbf{w}_{3}$ for some $\mathbf{w}_{1}, \mathbf{w}_{2}, \mathbf{w}_{3} \in \mathcal{X}^{*}$ such that the letter $y=\mathrm{h}\left(\mathbf{w}_{3}\right)$ is either

(a) simple in $\mathbf{w}$, or

(b) the first occurrence of some non-simple letter in w 
Hence $\mathbf{w}=\mathbf{w}_{1} x \mathbf{w}_{2} x y \mathbf{w}_{3}^{\prime}$ for some word $\mathbf{w}_{3}^{\prime}$. In both cases (a) and (b), the letter $y$ does not belong to the prefix $\mathbf{w}_{1} x \mathbf{w}_{2} x$. The connectedness of $\mathbf{w}$ implies the existence of a letter $h$ that is common to both $\mathbf{w}_{1} x \mathbf{w}_{2} x$ and $y \mathbf{w}_{3}^{\prime}$. Since the word $\mathbf{w}$ is quadratic, the letter $h$ is distinct from $x$ and $y$. Hence

$$
\mathbf{w} \stackrel{(\star)}{\approx} \mathbf{w}_{1} x \mathbf{w}_{2}\left(h x^{2} y h\right) \mathbf{w}_{3}^{\prime} \stackrel{(13)}{\approx} \mathbf{w}_{1} x \mathbf{w}_{2} h x y \times h \mathbf{w}_{3}^{\prime} \stackrel{(\star)}{\approx} \mathbf{w}_{1} x \mathbf{w}_{2} y x \mathbf{w}_{3}^{\prime}
$$

that is, the identities $\{(\star),(13)\}$ can be used to interchange the second occurrence of $x$ with the first letter $y$ of $\mathbf{w}_{3}$. Note that in this interchanging process,

- the distance between the two occurrences of $x$ is increased by one;

- if $y$ is non-simple, then the distance between the two occurrences of $y$ is increased by one;

- the distance between the two occurrences of any other non-simple letter remains unchanged;

- the resulting word is connected and is $\stackrel{\circ}{=}$-related to $\mathbf{w}$.

It is easy to see that the aforementioned process can be repeated on any letter that is not right-disciplined until it is right-disciplined. There are at most $|\mathbf{w}|$ ! words $\stackrel{\circ}{=}$-related to the word $\mathbf{w}$. Among all these $|\mathbf{w}|$ ! words, one must possess the greatest possible separation degree, say $d$. Hence the process of interchanging can only be repeated on $\mathbf{w}$ at most $d$ times. The resulting word $\mathbf{w}^{\delta}$ is then right-disciplined, connected, and quadratic such that $\mathbf{w} \stackrel{\circ}{=} \mathbf{w}^{\delta}$.

(ii) This is symmetrical to part (i).

(iii) By parts (i) and (ii), the deduction $\{(13),(14)\} \Vdash \mathbf{w} \approx \mathbf{w}^{\delta}$ holds for some quadratic, connected word $\mathbf{w}^{\delta}$ that is both left-disciplined and right-disciplined. The result now follows since $C_{0}^{1}(15)=C_{0}^{1}\{(13),(14)\}$ by Lemma 6.4.

(iv) Since the deduction (16) $\Vdash(15)$ holds by Lemma 6.4, it follows from part (iii) that the word $\mathbf{w}$ can be chosen to be disciplined, quadratic, and connected to begin with, say $\mathbf{w}=\mathbf{h w}^{\prime} \mathbf{t}$ is the word in (18). Since $\mathbf{w}$ is a connected word, it contains some non-simple letter $h$; this letter is clearly common to both the factors $\mathbf{h}$ and $\mathbf{t}$. Let $x$ and $y$ be any two consecutive letters in $\mathbf{w}^{\prime}$, say $\mathbf{w}^{\prime}=\mathbf{w}_{1} x y \mathbf{w}_{2}$ for some $\mathbf{w}_{1}, \mathbf{w}_{2} \in \mathcal{X}^{*}$. Since

$$
\mathbf{w} \stackrel{(\star)}{\approx} \mathbf{h w}_{1}(h x y h) \mathbf{w}_{2} \mathbf{t} \stackrel{(16)}{\approx} \mathbf{h w}_{1} h y \times h \mathbf{w}_{2} \mathbf{t} \stackrel{(\star)}{\approx} \mathbf{h w}_{1} y x \mathbf{w}_{2} \mathbf{t},
$$

the identities $\{(\star),(16)\}$ can be used to interchange the letters $x$ and $y$. It is easy to see how this can be repeated on the letters of the factor $\mathbf{w}^{\prime}$ until a well-disciplined word is obtained.

\section{Lemma 6.7.}

Let $\sigma: \mathbf{p}_{(\ell)} \mathbf{u q}_{(r)} \approx \mathbf{p}_{(\ell)} \mathbf{v} \mathbf{q}_{(r)}$ be the identity (4) that satisfies conditions (1†)-(6†) in Lemma 4.2. Suppose that the identity $\sigma$ is satisfied by the variety $A_{0} \vee N_{2}^{1}$ but not by the variety $B_{0}^{1}$. Then $C_{0}^{1}\{\sigma\}=C_{0}^{1}\left\{\theta, \sigma^{\prime}\right\}$ for some identity $\theta$ from $\{(13),(14),(15),(16)\}$ and some identity $\sigma^{\prime}$ of the variety $A_{0} \vee B_{0}^{1}$.

Proof. By condition (3†) of Lemma 4.2, the variety $\mathrm{N}_{2}^{1}$ satisfies the identity $\mathbf{u} \approx \mathbf{v}$. It follows from Lemma 2.1(iii) that

(a) the identity $\sigma$ does not delete to the identity $x^{2} \approx x$.

Let $\mathrm{V}=\mathrm{C}_{0}^{1}\{\sigma\}$. Then the variety $\mathrm{V}$ satisfies one of the four conditions in Corollary 6.5.

Case 1: $V \subseteq C_{0}^{1}(13)$ and $V \nsubseteq C_{0}^{1}(14)$. Then $V=C_{0}^{1}\{(13), \sigma\}$ and $\sigma \nVdash$ (14). By Lemma 6.6(i), the deduction (13) $\Vdash\left\{\mathbf{u} \approx \mathbf{u}^{\delta}, \mathbf{v} \approx \mathbf{v}^{\delta}\right\}$ holds for some right-disciplined, quadratic, connected words $\mathbf{u}^{\delta}$ and $\mathbf{v}^{\delta}$ such that $\mathbf{u} \doteq \mathbf{u}^{\delta}$ and $\mathbf{v} \stackrel{\circ}{=} \mathbf{v}^{\delta}$. Hence $\mathbf{V}=\mathrm{C}_{0}^{1}\left\{(13), \sigma^{\prime}\right\}$ where $\sigma^{\prime}$ is the identity $\mathbf{p}_{(\ell)} \mathbf{u}^{\delta} \mathbf{q}_{(r)} \approx \mathbf{p}_{(\ell)} \mathbf{v}^{\delta} \mathbf{q}_{(r)}$. By Lemma 4.1(iii), the identity $\sigma^{\prime}$ is satisfied by the variety $A_{0}$. Since $\mathbf{u} \stackrel{\circ}{=} \mathbf{u}^{\delta}$ and $\mathbf{v} \stackrel{\circ}{=} \mathbf{v}^{\delta}$, it follows from (a) that

(b) the identity $\sigma^{\prime}$ does not delete to the identity $x^{2} \approx x$. 
Recall that in a right-disciplined word $\mathbf{w}$, the second occurrences of non-simple letters of $\mathbf{w}$ form a suffix. Specifically, a right-disciplined word $\mathbf{w}$ cannot be of the form $\mathbf{a} x \mathbf{b} x \mathbf{c} y \mathbf{d}$ where $y$ is a simple letter in $\mathbf{w}$. Therefore, since $\mathbf{u}^{\delta}$ and $\mathbf{v}^{\delta}$ are right-disciplined words,

(c) the identity $\sigma^{\prime}$ does not delete to any identity in $\left\{x y x \approx x^{2} y, x^{2} y \approx y x^{2}\right\}$.

Suppose that the identity $\sigma^{\prime}$ deletes to some identity in $\left\{x y \approx y x, x y x \approx y x^{2}\right\}$. Then by Lemma 6.2, either the deduction $\sigma^{\prime} \Vdash(16)$ or the deduction $\sigma^{\prime} \Vdash(14)$ holds. It then follows from Lemma 6.4 that the inclusion $\mathrm{V} \subseteq \mathrm{C}_{0}^{1}(14)$ holds, contradicting the assumption of the present case. Therefore the identity $\sigma^{\prime}$ does not delete to any identity in $\left\{x y \approx y x, x y x \approx y x^{2}\right\}$. In the presence of (b), (c), and Lemma 2.2(ii), the identity $\sigma^{\prime}$ is satisfied by the variety $\mathrm{B}_{0}^{1}$.

Case 2: $\mathrm{V} \nsubseteq \mathrm{C}_{0}^{1}(13)$ and $\mathrm{V} \subseteq \mathrm{C}_{0}^{1}(14)$. It follows from an argument symmetrical to Case 1 that $\mathrm{V}=\mathrm{C}_{0}^{1}\left\{(14)\right.$, $\left.\sigma^{\prime}\right\}$ for some identity $\sigma^{\prime}$ of the variety $\mathrm{B}_{0}^{1}$.

Case 3: $\mathrm{V} \subseteq \mathrm{C}_{0}^{1}(15)$ and $\mathrm{V} \nsubseteq \mathrm{C}_{0}^{1}(16)$. Then $\mathrm{V}=\mathrm{C}_{0}^{1}\{(15), \sigma\}$ and $\sigma \nVdash$ (16). By Lemma 6.6(iii), the deduction (15) $\Vdash\left\{\mathbf{u} \approx \mathbf{u}^{\delta}, \mathbf{v} \approx \mathbf{v}^{\delta}\right\}$ holds for some disciplined, quadratic, connected words $\mathbf{u}^{\delta}$ and $\mathbf{v}^{\delta}$ such that $\mathbf{u} \doteq \mathbf{u}^{\delta}$ and $\mathbf{v} \doteq \mathbf{v}^{\delta}$. Hence $\mathbf{V}=\mathrm{C}_{0}^{1}\left\{(15), \sigma^{\prime}\right\}$ where $\sigma^{\prime}$ is the identity $\mathbf{p}_{(\ell)} \mathbf{u}^{\delta} \mathbf{q}_{(r)} \approx \mathbf{p}_{(\ell)} \mathbf{v}^{\delta} \mathbf{q}_{(r)}$. By Lemma 4.1(iii), the identity $\sigma^{\prime}$ is satisfied by the variety $A_{0}$. Since $\mathbf{u} \stackrel{\circ}{=} \mathbf{u}^{\delta}$ and $\mathbf{v} \stackrel{\circ}{=} \mathbf{v}^{\delta}$, it follows from (a) that

(d) the identity $\sigma^{\prime}$ does not delete to the identity $x^{2} \approx x$.

A disciplined word $\mathbf{w}$ cannot be of the form $\mathbf{a} x \mathbf{b} \mathbf{b} \mathbf{c} y \mathbf{d}$ or $\mathbf{a} y \mathbf{b} x \mathbf{c} x \mathbf{d}$ where $y$ is a simple letter in $\mathbf{w}$. Therefore, since $\mathbf{u}^{\delta}$ and $\mathbf{v}^{\delta}$ are disciplined words,

(e) the identity $\sigma^{\prime}$ does not delete to any identity in $\left\{x y x \approx x^{2} y, x y x \approx y x^{2}, x^{2} y \approx y x^{2}\right\}$.

Suppose that the identity $\sigma^{\prime}$ deletes to the identity $x y \approx y x$. Then the deduction $\sigma^{\prime} \Vdash(16)$ holds by Lemma 6.2. It follows that the inclusion $\mathrm{V} \subseteq \mathrm{C}_{0}^{1}(16)$ holds, contradicting the assumption of the present case. Therefore the identity $\sigma^{\prime}$ does not delete to the identity $x y \approx y x$. In the presence of (d), (e), and Lemma 2.2(ii), the identity $\sigma^{\prime}$ is satisfied by the variety $\mathrm{B}_{0}^{1}$.

Case 4: $\mathrm{V} \subseteq \mathrm{C}_{0}^{1}(16)$. Then $\mathrm{V}=\mathrm{C}_{0}^{1}\{(16), \sigma\}$. By Lemma 6.6(iv), the deduction (16) $\Vdash\left\{\mathbf{u} \approx \mathbf{u}^{\delta}, \mathbf{v} \approx \mathbf{v}^{\delta}\right\}$ holds for some well-disciplined, quadratic, connected words $\mathbf{u}^{\delta}$ and $\mathbf{v}^{\delta}$ such that $\mathbf{u} \doteq \mathbf{u}^{\delta}$ and $\mathbf{v} \doteq \mathbf{v}^{\delta}$. Therefore $\mathbf{V}=\mathrm{C}_{0}^{1}\left\{(16), \sigma^{\prime}\right\}$ where $\sigma^{\prime}$ is the identity $\mathbf{p}_{(\ell)} \mathbf{u}^{\delta} \mathbf{q}_{(r)} \approx \mathbf{p}_{(\ell)} \mathbf{v}^{\delta} \mathbf{q}_{(r)}$. By Lemma 4.1 (iii), the identity $\sigma^{\prime}$ is satisfied by the variety $\mathrm{A}_{0}$. Since $\mathbf{u} \stackrel{\circ}{=} \mathbf{u}^{\delta}$ and $\mathbf{v} \stackrel{\circ}{=} \mathbf{v}^{\delta}$, it follows from (a) that the identity $\sigma^{\prime}$ does not delete to the identity $x^{2} \approx x$. Since $\mathbf{u}^{\delta}$ and $\mathbf{v}^{\delta}$ are well-disciplined words, the identity does not delete to any identity from (2). Hence by Lemma 2.2(ii), the identity $\sigma^{\prime}$ is satisfied by the variety $B_{0}^{1}$.

\section{Proposition 6.8.}

Let $\mathrm{V}$ be any variety in the interval $\left[\mathrm{A}_{0} \vee \mathrm{N}_{2}^{1}, \mathrm{C}_{0}^{1}\right]$ such that $B_{0}^{1} \notin \mathrm{V}$. Then the variety $\mathrm{V}$ is finitely based.

Proof. By assumption, $\mathrm{V}=\mathrm{C}_{0}^{1} \Sigma$ for some set $\Sigma$ of identities of the variety $\mathrm{A}_{0} \vee \mathrm{N}_{2}^{1}$. By Lemma 4.2, the identities in the set $\Sigma$ can be chosen to be of the form (4) that satisfy conditions (1†)-(6†). Let $\Sigma=\Sigma_{0} \cup \Sigma_{1}$ where $\Sigma_{1}$ is the set of all identities from $\Sigma$ that are satisfied by the variety $B_{0}^{1}$ and $\Sigma_{0}=\Sigma \backslash \Sigma_{1}$. Then it follows from Lemma 6.7 that $C_{0}^{1} \Sigma_{0}=C_{0}^{1}\left(\Theta \cup \Sigma_{0}^{\prime}\right)$ for some set $\Theta$ of identities from $\{(13),(14),(15),(16)\}$ and some set $\Sigma_{0}^{\prime}$ of identities of the variety $A_{0} \vee B_{0}^{1}$. Since the identities in $\Sigma_{0}^{\prime}$ and $\Sigma_{1}$ are satisfied by the variety $A_{0} \vee B_{0}^{1}$, the variety $C_{0}^{1}\left(\Sigma_{0}^{\prime} \cup \Sigma_{1}\right)$ belongs to the interval $\left[A_{0} \vee B_{0}^{1}, C_{0}^{1}\right]$ and is finitely based by Proposition 5.1. Consequently, the variety $\vee=C_{0}^{1} \Theta \cap C_{0}^{1}\left(\Sigma_{0}^{\prime} \cup \Sigma_{1}\right)$ is finitely based.

\subsection{Varieties in $\left[A_{0}, C_{0}^{1}\right]$ not containing $N_{2}^{1}$.}

The main result of this subsection, Proposition 6.11, establishes the finite basis property of every variety in the interval $\left[A_{0}, C_{0}^{1}\right]$ that does not contain the variety $N_{2}^{1}$. 


\section{Lemma 6.9.}

Let $\mathrm{V}$ be any variety in the interval $\left[\mathrm{A}_{0}, \mathrm{C}_{0}^{1}\right]$. If $\mathrm{N}_{2}^{1} \notin \mathrm{V}$, then the variety $\mathrm{V}$ satisfies the identity

$$
h x^{2} h \approx h x h
$$

Proof. By Remark 2.4 and Lemmas 2.1(iii) and 4.1, the variety $\mathbf{V}$ satisfies some quadratic identity $\mathbf{u} \approx \mathbf{v}$ such that $\operatorname{con}(\mathbf{u})=\operatorname{con}(\mathbf{v})$ and $\mathbf{u} \neq \mathbf{v}$. Then the identity $\mathbf{u} \approx \mathbf{v}$ deletes to the identity $x^{2} \approx x$. By Lemma 6.2 , the variety $\mathbf{V}$ satisfies the identity (19).

\section{Lemma 6.10.}

Let $\sigma: \mathbf{p}_{(\ell)} \mathbf{u} \mathbf{q}_{(r)} \approx \mathbf{p}_{(\ell)} \mathbf{v} \mathbf{q}_{(r)}$ be the identity (4) that satisfies conditions (1†)-(6†) in Lemma 4.2. Suppose that the identity $\sigma$ is not satisfied by the variety $\mathrm{N}_{2}^{1}$. Then $\mathrm{C}_{0}^{1}\{\sigma\}=\mathrm{C}_{0}^{1}\left\{(19), \sigma^{\prime}\right\}$ for some identity $\sigma^{\prime}$ of the variety $\mathrm{A}_{0} \vee \mathrm{N}_{2}^{1}$.

Proof. It follows from Lemma 6.9 that $\mathrm{C}_{0}^{1}\{\sigma\}=\mathrm{C}_{0}^{1}\{(19), \sigma\}$. Note that if $x$ is any simple letter in the connected word $\mathbf{u}$, say $\mathbf{u}=\mathbf{u}_{1} x \mathbf{u}_{2}$ where the factors $\mathbf{u}_{1}$ and $\mathbf{u}_{2}$ share some common letter $h$, then

$$
\mathbf{u} \stackrel{(\star)}{\approx} \mathbf{u}_{1}(h x h) \mathbf{u}_{2} \stackrel{(19)}{\approx} \mathbf{u}_{1} h x^{2} h \mathbf{u}_{2} \stackrel{(\star)}{\approx} \mathbf{u}_{1} x^{2} \mathbf{u}_{2}
$$

Similarly, the identities $\{(\star),(19)\}$ can be used to replace any simple letter $x$ in $\mathbf{v}$ by $x^{2}$. Let $\mathbf{u}^{\prime}$ and $\mathbf{v}^{\prime}$ be the words obtained from the words $\mathbf{u}$ and $\mathbf{v}$ by replacing every simple letter $x$ by $x^{2}$, and let $\sigma^{\prime}$ be the identity $\mathbf{p}_{(\ell)} \mathbf{u}^{\prime} \mathbf{q}_{(r)} \approx \mathbf{p}_{(\ell)} \mathbf{v}^{\prime} \mathbf{q}_{(r)}$. Then $\mathrm{C}_{0}^{1}\{\sigma\}=\mathrm{C}_{0}^{1}\{(19), \sigma\}=\mathrm{C}_{0}^{1}\left\{(19), \sigma^{\prime}\right\}$. By conditions (2†) and (4†) of Lemma 4.2, the identity $\mathbf{u} \approx \mathbf{v}$ is satisfied by the variety $A_{0}$ and the words $\mathbf{u}$ and $\mathbf{v}$ are connected. Clearly $\mathbf{u}^{\prime}$ and $\mathbf{v}^{\prime}$ are connected, quadratic words that contain no simple letters. By Lemmas 2.1 (iii) and 4.1, the identity $\mathbf{u}^{\prime} \approx \mathbf{v}^{\prime}$ is satisfied by the variety $A_{0} \vee N_{2}^{1}$. Therefore the identity $\sigma^{\prime}$ is also satisfied by the variety $A_{0} \vee N_{2}^{1}$.

\section{Proposition 6.11.}

Let $\mathrm{V}$ be any variety in the interval $\left[\mathrm{A}_{0}, \mathrm{C}_{0}^{1}\right]$ such that $\mathrm{N}_{2}^{1} \notin \mathrm{V}$. Then the variety $\mathrm{V}$ is finitely based.

Proof. It follows from Lemma 6.9 that $\mathrm{V}=\mathrm{C}_{0}^{1}((19) \cup \Sigma)$ for some set $\Sigma$ of identities of the variety $\mathrm{A}_{0}$. By Lemma 4.2, the identities in the set $\Sigma$ can be chosen to be of the form (4) with conditions (1†)-(6†). Let $\Sigma=\Sigma_{0} \cup \Sigma_{1}$ where $\Sigma_{1}$ is the set of all identities from $\Sigma$ that are satisfied by the variety $N_{2}^{1}$ and $\Sigma_{0}=\Sigma \backslash \Sigma_{1}$. Then it follows from Lemma 6.10 that $C_{0}^{1}\left((19) \cup \Sigma_{0}\right)=C_{0}^{1}\left((19) \cup \Sigma_{0}^{\prime}\right)$ for some set $\Sigma_{0}^{\prime}$ of identities of the variety $A_{0} \vee N_{2}^{1}$. Since the identities in $\Sigma_{0}^{\prime}$ and $\Sigma_{1}$ are satisfied by the variety $A_{0} \vee N_{2}^{1}$, the variety $V^{\prime}=C_{0}^{1}\left(\Sigma_{0}^{\prime} \cup \Sigma_{1}\right)$ belongs to the interval $\left[A_{0} \vee N_{2}^{1}, C_{0}^{1}\right]$. If $B_{0}^{1} \notin V^{\prime}$, then the variety $\mathrm{V}^{\prime}$ is finitely based by Proposition 6.8. If $B_{0}^{1} \in \mathrm{V}^{\prime}$, then the variety $\mathrm{V}^{\prime}$ is finitely based by Proposition 5.1. In any case, the variety $\mathrm{V}=\mathrm{C}_{0}^{1}(19) \cap \mathrm{V}^{\prime}$ is finitely based.

\section{Acknowledgements}

The author is very grateful to the referees for suggestions that improved the presentation of the article.

\section{References}

[1] Almeida J., Finite Semigroups and Universal Algebra, Series in Algebra, 3, World Scientific, Singapore, 1994

[2] Burris S., Sankappanavar H.P., A Course in Universal Algebra, Grad. Texts in Math., 78, Springer, New YorkHeidelberg-Berlin, 1981 
[3] Edmunds C.C., On certain finitely based varieties of semigroups, Semigroup Forum, 1977, 15(1), 21-39

[4] Edmunds C.C., Varieties generated by semigroups of order four, Semigroup Forum, 1980, 21(1), 67-81

[5] Hall T.E., Kublanovskii S.I., Margolis S., Sapir M.V., Trotter P.G., Algorithmic problems for finite groups and finite 0-simple semigroups, J. Pure Appl. Algebra, 1997, 119(1), 75-96

[6] Lee E.W.H., Identity bases for some non-exact varieties, Semigroup Forum, 2004, 68(3), 445-457

[7] Lee E.W.H., Subvarieties of the variety generated by the five-element Brandt semigroup, Internat. J. Algebra Comput., 2006, 16(2), 417-441

[8] Lee E.W.H., On identity bases of exclusion varieties for monoids, Comm. Algebra, 2007, 35(7), 2275-2280

[9] Lee E.W.H., Combinatorial Rees-Sushkevich varieties are finitely based, Internat. J. Algebra Comput., 2008, 18(5), 957-978

[10] Lee E.W.H., On the variety generated by some monoid of order five, Acta Sci. Math. (Szeged), 2008, 74(3-4), 509-537

[11] Lee E.W.H., Hereditarily finitely based monoids of extensive transformations, Algebra Universalis, 2009, 61(1), 31-58

[12] Lee E.W.H., Combinatorial Rees-Sushkevich varieties that are Cross, finitely generated, or small, Bull. Aust. Math. Soc., 2010, 81(1), 64-84

[13] Lee E.W.H., Varieties generated by 2-testable monoids (submitted)

[14] Lee E.W.H., Li J.R., Minimal non-finitely based monoids (submitted)

[15] Lee E.W.H., Volkov M.V., On the structure of the lattice of combinatorial Rees-Sushkevich varieties, In: Semigroups and Formal Languages, Lisboa, 2005, World Scientific, Singapore, 2007, 164-187

[16] Lee E.W.H., Volkov M.V., Limit varieties generated by completely 0-simple semigroups, Internat. J. Algebra Comput. (in press)

[17] Luo Y.F., Zhang W.T., On the variety generated by all semigroups of order three (submitted)

[18] Perkins P., Bases for equational theories of semigroups, J. Algebra, 1969, 11(2), 298-314

[19] Sapir M.V., Problems of Burnside type and the finite basis property in varieties of semigroups, Math. USSR, Izv., 1988, 30(2), 295-314

[20] Shevrin L.N., Volkov M.V., Identities of semigroups, Soviet Math. (Iz. VUZ), 1985, 29(11), 1-64

[21] Torlopova N.G., Varieties of quasi-orthodox semigroups, Acta Sci. Math. (Szeged), 1984, 47(3-4), $297-301$ (in Russian)

[22] Trahtman A.N., The finite basis question for semigroups of order less than six, Semigroup Forum, 1983, 27(1), 387-389

[23] Trahtman A.N., Some finite infinitely basable semigroups, Ural. Gos. Univ. Mat. Zap., 1987, 14(2), $128-131$ (in Russian)

[24] Trahtman A.N., Identities of a five-element 0-simple semigroup, Semigroup Forum, 1994, 48(3), 385-387

[25] Trahtman A.N., Identities of locally testable semigroups, Comm. Algebra, 1999, 27(11), 5405-5412

[26] Volkov M.V., The finite basis question for varieties of semigroups, Math. Notes, 1989, 45(3), 187-194

[27] Volkov M.V., The finite basis problem for finite semigroups, Sci. Math. Jpn., 2001, 53(1), 171-199

[28] Zhang W.T., Some Studies on Varieties Generated by Finite Semigroups and Their Subvarieties Lattices, PhD thesis, Lanzhou University, Gansu, China, 2009 (in Chinese) 